\title{
Modeling reaction time and accuracy of multiple-alternative decisions
}

\author{
FÁBio P. LEITE \\ Ohio State University, Lima, Ohio \\ AND \\ ROGER RATCLIFF \\ Ohio State University, Columbus, Ohio
}

\begin{abstract}
Several sequential-sampling models using racing diffusion processes for multiple-alternative decisions were evaluated, using data from two perceptual discrimination experiments. The structures of the models differed on a number of dimensions, including whether there was lateral inhibition between accumulators, whether there was decay in evidence, whether evidence could be negative, and whether there was variability in starting points. Data were collected from a letter discrimination task in which stimulus difficulty and probability of the response alternatives were varied along with number of response alternatives. Model-fitting results ruled out a large number of model classes in favor of a smaller number of specific models, most of which showed a moderate to high degree of mimicking. The best-fitting models had zero to moderate values of decay, had no inhibition, and assumed that the addition of alternatives affected the subprocesses contributing to the nondecisional time, the degree of caution, or the quality of evidence extracted from stimuli.
\end{abstract}

Perceptual decision making is an area of research that has received a great deal of attention over the last 10 years or so. In psychology, it has been investigated with a range of approaches, from experimental to theoretical (Bogacz, Usher, Zhang, \& McClelland, 2007; Ratcliff \& Rouder, 1998; Ratcliff, Van Zandt, \& McKoon, 1999; P. L. Smith, 1995; P. L. Smith \& Ratcliff, 2009; P. L. Smith, Ratcliff, \& Wolfgang, 2004; Usher \& McClelland, 2001), and it has been studied with combined theoretical and empirical approaches in neuroscience (Gold \& Shadlen, 2000; Newsome, Britten, \& Movshon, 1989; Salzman \& Newsome, 1994; Shadlen \& Newsome, 2001; Supèr, Spekreijse, \& Lamme, 2001). In most research to date, the focus has been on the two-choice experimental paradigm (e.g., Ratcliff \& Rouder, 1998). There has also been an accumulating body of research that has taken models of processing and extended them to multiple-choice paradigms (Bogacz et al., 2007; McMillen \& Holmes, 2006; Usher \& McClelland, 2004; Usher, Olami, \& McClelland, 2002). But to this point in time, there have been relatively few combined experimental and theoretical studies of multiplealternative perceptual decision making. Our aim in this article is to address the lack of such studies by presenting an experiment and comprehensive theoretical analyses. ${ }^{1}$

The growing consensus in the perceptual-decisionmaking domain is that only models that assume that evidence is gradually accumulated over time can account for the full range of experimental data - namely, accuracy and both correct and error reaction time (RT) distributions.
Two variants of this general class are the Wiener diffusion process model (Ratcliff, 1978, 2002; Ratcliff \& McKoon, 2008; Ratcliff \& Rouder, 2000) and the multiple racing diffusion processes model (Ratcliff, 2006; P. L. Smith, 2000; Usher \& McClelland, 2001). In the standard diffusion process, evidence is accumulated in a single variable toward one of two decision criteria. This model is difficult to extend to multiple alternatives, although Laming (1968) and Pike (1966), for example, have offered qualitative suggestions. The model that seems most natural for the multiple-alternative paradigm assumes that evidence is accumulated in separate accumulators, corresponding to the different alternatives. In particular, the model that best exemplifies the set of features we wish to test is the leaky competing accumulator (LCA; Usher \& McClelland, 2001). This model assumes that stochastic accumulation of information occurs continuously over time, with leakage (decay) and lateral inhibition (competition among accumulators), with the possibility of variability in both starting point and the drift rates driving the accumulation process. The LCA model, however, has been fit to relatively few experimental data sets.

The general evidence accumulation model has been applied to a number of domains, from neurophysiological data to cognitive tasks such as memory, lexical processing, and absolute identification, to aging and impaired processing, and to consumer decision making (Boucher, Palmeri, Logan, \& Schall, 2007; Brown \& Heathcote, 2005; Brown, Marley, Donkin, \& Heathcote, 
2008; Busemeyer \& Townsend, 1993; Ditterich, 2006; Gomez, Perea, \& Ratcliff, 2007; Mazurek, Roitman, Ditterich, \& Shadlen, 2003; Niwa \& Ditterich, 2008; Ratcliff, Cherian, \& Segraves, 2003; Ratcliff, Gomez, \& McKoon, 2004; Ratcliff, Hasegawa, Hasegawa, Smith, \& Segraves, 2007; Ratcliff \& Smith, 2004; Ratcliff, Thapar, \& McKoon, 2006; Ratcliff, Thapar, Smith, \& McKoon, 2005; Ratcliff \& Van Dongen, 2009; Roe, Busemeyer, $\&$ Townsend, 2001). One of the features of models in the evidence accumulation class is that in order for them to successfully account for the full range of experimental data, they need to assume that various components of processing vary from trial to trial (Laming, 1968; Ratcliff, 1978; Ratcliff \& Rouder, 1998; Ratcliff et al., 1999). These models can be contrasted with signal detection theory (SDT; Swets, Tanner, \& Birdsall, 1961), in which all sources of noise are combined into a single source-namely, variability in perceptual strength. For example, in the class of diffusion process models, the decision process is assumed to be variable, within a trial (within-trial noise) and across trials, in perceptual strength (drift rate) and starting points.

In neuroscience, Hanes and Schall (1996) were the first to convincingly argue that it is possible to relate evidence accumulation models to single-cell recording data. They suggested that rhesus monkeys' saccadic movements were initiated if and only if the neural activity in frontal eye field cells surpassed a (constant) threshold and that RT distribution was a resultant of the stochastic variability in the rate at which neural activity grew toward that threshold. Following Hanes and Schall's work, neurobiology in the decision-making field seems to have adopted the motion discrimination paradigm to examine perceptual decision making (e.g., Ditterich, 2006; Gold \& Shadlen, 2000; Heekeren, Marrett, Ruff, Bandettini, \& Ungerleider, 2006; Niwa \& Ditterich, 2008; Palmer, Huk, \& Shadlen, 2005; Ratcliff \& McKoon, 2008; Roitman \& Shadlen, 2002; Salzman \& Newsome, 1994; Shadlen \& Newsome, 2001). In a standard motion discrimination task, a percentage of dots in a display move coherently, while the remaining dots are shuffled to random positions. Primate or human participants decide in which direction the coherent dots are moving and respond with a saccade to a target or with a keypress.

In several studies, models that assumed accumulation of information toward decision criteria have been applied to experimental data from the motion discrimination task. In some of these studies (Mazurek et al., 2003; Palmer et al., 2005; Roitman \& Shadlen, 2002), joint RT and accuracy were collected, but the models were either not fit to the full range of experimental data or fit relatively poorly. Because the models were not successfully fit to the range of behavioral data, it is not possible to determine whether the models need the various sources of variability in processing to account for the full range of data. Nevertheless, some of the models used only within-trial noise. Models in Ditterich (2006), Niwa and Ditterich (2008), and Ratcliff and McKoon (2008), on the other hand, attempted to fit accuracy and RT distributions for both correct and error responses jointly and, so, identify the different sources of noise.

Evidence accumulation models have also been related to physiological measures in humans, using both functional magnetic resonance imaging (fMRI) and electroencephalography (EEG). Heekeren et al. (2006), for example, found evidence for a decision variable existing independently of motor planning and execution. They had participants express their decision about direction of motion using two independent motor systems, oculomotor and manual, and found that four brain regions showed an increased BOLD signal to high coherence (relative to low coherence), independent of the motor system used to express the decision. Philiastides, Ratcliff, and Sajda (2006), using a single-trial analysis of EEG data from a face-car discrimination task with human participants, found support for a time separation between perceptual processing and decision-making processing. This separation suggests that cortical networks could dynamically allocate additional processing time for difficult decisions.

In behavioral research in psychology, perceptual decision making has been studied using models that make a great deal of contact between theory and data. In particular, the sequential sampling framework has been successful in accounting for both RT and accuracy, as well as speed-accuracy trade-off effects (for reviews, see Luce, 1986; Ratcliff \& Smith, 2004; Vickers, Caudrey, \& Willson, 1971), continuing to be of critical theoretical interest. An exemplar of this particular modeling approach is the diffusion model proposed by Ratcliff (1978). Using a numerosity judgment task, for example, Ratcliff et al. (1999) showed that the diffusion model could explain how both correct and incorrect decisions are made, how their relative speeds change as a function of experimental conditions, and why RT distributions have their characteristic shapes. Models implementing multiple racing diffusion processes (e.g., Bogacz et al., 2007; Ratcliff et al., 2007; Usher \& McClelland, 2001) not only extend well to multiple-alternative paradigms, but also qualitatively fit two-choice data as well as the diffusion model (Ratcliff, 2006; Ratcliff \& Smith, 2004; Ratcliff et al., 2005).

The evidence accumulation models have a major advantage over static models of decision making, in that they allow for several sources of variability that occur in different components of processing and that are identifiable (Ratcliff \& McKoon, 2008; Ratcliff \& Tuerlinckx, 2002). For example, there can be variability in perceptual strength (variability in drift rate across trials), in starting points of the process or decision criteria, in the decision process itself (within trial noise), and in the duration of other processes (encoding and response output). The ability of these models to identify such variability sources is a major advance because they allow noise in processing to be separated into sources that occur at different points in the stream of processing, from encoding to decision. If our experimental and theoretical work is to be related to physiological measures (i.e., EEG, fMRI, magnetoencephalography, or single-cell recording), we need the 
ability to separate different sources of variability (e.g., Ratcliff, Philiastides, \& Sajda, 2009).

Our aim in this article is to present experiments in which the number of alternatives was manipulated along with the difficulty of the decision. This allows us to test whether the data support one or many of a range of possible model features. What we aim to learn about perceptual decision making from this study is whether these various architectural features are necessary. In particular, is lateral inhibition between alternatives needed? Does the evidence in the accumulators decay the more evidence is accumulated? In addition, the modeling will allow us to determine which sources of variability play an important role in processing.

We present empirical data from a multiple-alternative paradigm and report tests of a number of racing diffusion process models that differ on a number of dimensions, with the various combinations of these dimensions leading to 384 possible models. We exclude most of them in preliminary analyses and report fitting details of 16 models. More specifically, we report data collected from two experiments involving a letter discrimination task in which two, three, or four response alternatives were used. The difficulty of the decision was manipulated in the first experiment by changing the discriminability of the stimuli and, in the second, by varying the proportion of stimuli corresponding to the different responses. Subsequently, we describe what properties were found in the best-fitting models and examine the impact of increasing the number of alternatives on the two manipulations (difficulty and proportion).

\section{Hick's Law}

In early investigations of the impact of increasing the number of alternatives, Hick (1952) and Hyman (1953) described evidence that showed that mean reaction time (MRT) should increase with number of alternatives. If the stimuli have equal probability, this is currently viewed as a well-established fact (e.g., Luce, 1986). Hick further noted that this relationship was best fit by

$$
\mathrm{MRT}=A+B \log (n+1) .
$$

Several studies following Hick's (1952) article presented results that conformed to Equation 1 or to a variant, replacing $(n+1)$ with $n$, both commonly referred to as Hick's law or as the Hick-Hyman law (see Welford, 1980, pp. 73-77, for a survey of the original Hick's law and variants). Models of the type we evaluated are capable of producing MRTs that conform to Hick's law. In free response protocols, for example, McMillen and Holmes (2006) showed that leaky accumulator (LA) models with absolute threshold implementations perform nearly optimally (by minimizing the decision time for a predetermined level of accuracy) for moderate (and approximately equal) values of decay and inhibition and error rates larger than $10 \%$. Under these circumstances, predicted MRTs conform to Hick's law, and this correspondence is found so long as all accumulators in the model receive equal levels of noise (see also Bogacz et al., 2007). The models reported in what follows all assume equal levels of noise for all accumulators.

Parenthetically, by defining MRT in terms of the signalto-noise ratio $a / c$ (drift rate over the square root of the diffusion coefficient), optimal performance is achieved when

$$
\begin{aligned}
\operatorname{MRT}=\left(\frac{c}{a}\right)^{2}\left\{\log \left(\frac{1}{\mathrm{ER}}\right)+\frac{\left(h_{n-1}^{*}\right)^{2}}{2}\right. & \left.\cdot\left[1+\sqrt{1+\frac{4}{\left(h_{n-1}^{*}\right)^{2}} \log \left(\frac{1}{\mathrm{ER}}\right)}\right]\right\}+o(1),
\end{aligned}
$$

where $h_{n-1}^{*}$ is the expected value of the $(n-1)$ th standard normal order statistic and ER is the error rate (cf. McMillen \& Holmes, 2006, Equation 37).

\section{Models}

Sequential-sampling models have been developed and successfully applied to cognitive tasks, but they usually involve two alternatives. For this project, we investigated multiple-alternative extensions for some members of the sequential-sampling family of models. In 2004, Ratcliff and Smith reported an evaluation of four widely used sequential-sampling models: the Wiener diffusion model (Laming, 1968; Link, 1975; Ratcliff, 1978, 1981, 1985, 1988a), the Ornstein-Uhlenbeck (OU) diffusion model (Busemeyer \& Townsend, 1992, 1993; P. L. Smith, 2000), Vickers's accumulator model (LaBerge, 1962; P. L. Smith \& Vickers, 1988; Vickers, 1970), and the Poisson counter model (P. L. Smith \& Van Zandt, 2000; Townsend \& Ashby, 1983). Although the Wiener diffusion process model produced the best fits to the two-choice RT data from three experiments against which these four models were tested, an extension of this model to multiplealternative paradigms is not straightforward. However, Ratcliff and Smith also reported that three other models, whose architectures are suitable to multiple alternatives, mimicked the Wiener diffusion process model and produced fits almost as good as that of the Wiener diffusion process model. These models were Usher and McClelland's (2001) LCA model and two other models assuming racing diffusion processes, either with absolute stopping criteria and decay or with relative stopping criteria (i.e., max vs. next; for reports of similar results, see Ratcliff, 2006; Ratcliff et al., 2005).

Usher and McClelland's (2001) LCA model assumes that stochastic accumulation of information occurs continuously over time, with leakage (decay) and lateral inhibition. There is one accumulator implementing a diffusion process for each response alternative, and a response is made when an accumulator reaches its respective decision criterion (see Figure 1). The rate at which one accumulator approaches its criterion is determined by the input from the stimulus $(\rho)$, the decay $(\kappa)$, and the lateral inhibition $(\beta)$. Inhibition increases as the amount of information increases in the other accumulators, and the 


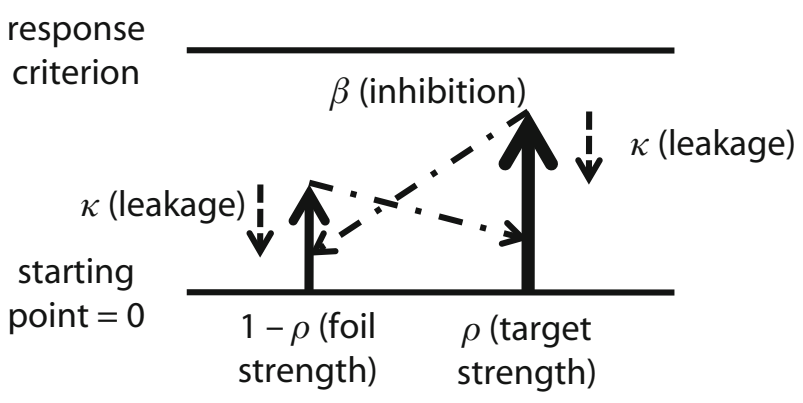

Figure 1. The leaky competing accumulator model with two accumulators. Free parameters of the model are criteria $\left(c_{1}\right.$ and $\left.c_{2}\right)$, decay $(\kappa)$, inhibition $(\beta)$, noise in the accumulation process $(\sigma)$, starting point (sp), and input strength $(\rho)$. One could also include range of variability in starting point $\left(s_{\mathrm{sp}}\right)$, a nondecisional component $\left(t_{\mathrm{er}}\right)$, and variability in $t_{\mathrm{er}}\left(s_{t}\right)$.

size of decay increases as information in the accumulator increases. Accuracy and RT are modeled simultaneously, and the growth of evidence in the accumulators is governed by

$$
d x_{i}=\left[\rho_{i}-\kappa x_{i}-\beta \sum_{j \neq i} x_{j}\right] \frac{d t}{\tau}+\xi_{i} \sqrt{\frac{d t}{\tau}},
$$

where $x_{i}$ takes $\max \left(x_{i}, 0\right)$-remaining nonnegative — and $\xi$ represents standard deviation in noise in the accumulation process (cf. Usher \& McClelland, 2001, Equation 4). As in Usher and McClelland, in our implementations of this model and other variants, $\tau$ was set such that $d t / \tau$ corresponded to $10-\mathrm{msec}$ steps. Input strength parameters were constrained so that $\Sigma_{i} \rho_{i}=1$.

In order for the LCA model to predict errors faster than correct responses (e.g., in very easy conditions), Usher and McClelland (2001) assumed rectangularly distributed (with zero minima) starting points for the accumulators, following Laming (1968) and Ratcliff et al. (1999). In all the racing diffusion process models we considered, nondecisional components (e.g., stimulus encoding and motor response processes) are combined into a single random variable, $t_{\mathrm{er}}$, which is assumed to vary across trials. In the models, this nondecisional component takes values from a rectangular distribution with mean $T_{\text {er }}$ and range $s_{t}$, and the predicted MRT is the mean time it takes the decision process to terminate plus the nondecisional component.

Models for fitting the data. There are 384 racing diffusion process models we could produce from all possible combinations of the assumptions we presented above. We summarize the various theoretical options below (which have all been used or proposed previously in the literature), followed by brief discussions about the theoretical issues of process or representation associated with them within the framework of the LCA model.

1. Decaying versus nondecaying accumulation. For $\kappa>0$ in Equation 3, evidence in any accumulator decays by an amount proportional to the amount of evidence in it. The presence or absence of decay is chiefly an architectural issue. As was reported in P. L. Smith (1995), the spontane- ous decay of some proportion of the accumulated signal inherently bounds the sensitivity of the decision stage.

2. Competing versus independent accumulation. For $\beta>0$ in Equation 3, accumulation of evidence to any accumulator is inhibited in proportion to the amount of evidence accumulated at this point by its competitors. The assumption that one alternative influences the accumulation of evidence for competing alternatives is a plausible processing assumption discussed by Usher and McClelland (2001), who noted that the use of lateral inhibition enables the emulation of relative-evidence diffusion processes while using an absolute threshold criterion, allowing the model to be applied equivalently to two- and multiple-choice paradigms (p. 552).

3. Starting-point variability versus identical starting points. Adding variability in starting points allows random initial biases toward one of the alternatives (e.g., Laming, 1968), making it a crucial feature in accounting for fast error responses in the data. Variability in starting point can be modeled in two ways. In its simplest form, each accumulator starts from a random point, drawn from a uniform distribution between 0 and a limit determined by a free parameter $\left(s_{\mathrm{sp}}\right)$. In an alternative form, starting points are negatively correlated, as in Ratcliff et al. (2007). In the latter case, a number $x$ is randomly selected from a uniform distribution between 0 and $s_{\mathrm{sp}} / 2$; one accumulator is set to start at $\left(s_{\mathrm{sp}} / 2+x\right)$, and the other accumulators start at $\left(s_{\mathrm{sp}} / 2-x\right)$.

4. Unbounded versus bounded evidence accumulation. In racing accumulator models, it is often assumed that activity cannot fall below zero. This assumption is usually justified by appeal to neural plausibility, but from a mathematical point of view, one could relax the bounded evidence accumulation assumption by allowing accumulators to take on negative values.

5. One nondecisional-component parameter or one parameter for each number of alternatives. As was noted above, all the models we considered assumed that the durations of all components of processing other than the decision component are combined into a single random variable that varies across trials. This nondecisional component is commonly assumed to include encoding and motor-response times. As more alternatives are added, it is possible that some motor component, such as motor preparation, slows down. Thus, the nondecisional component can be assumed to differ with number of alternatives (i.e., two, three, or four alternatives), resulting in three additional free parameters in the model. Or it can be the same across number of alternatives, adding a single free parameter to the model.

6. One versus three decision criterion parameters. Models can have either one single value for all the decision criteria for all numbers of alternatives or different parameters, one for each number of alternatives involved in the decision. Psychologically, it is plausible to have a higher degree of caution in conditions involving higher numbers of alternatives.

7. Target-foil versus accumulator-specific input strength parameters. Two assumptions regarding input strength parameters can be made. First, the letter corre- 


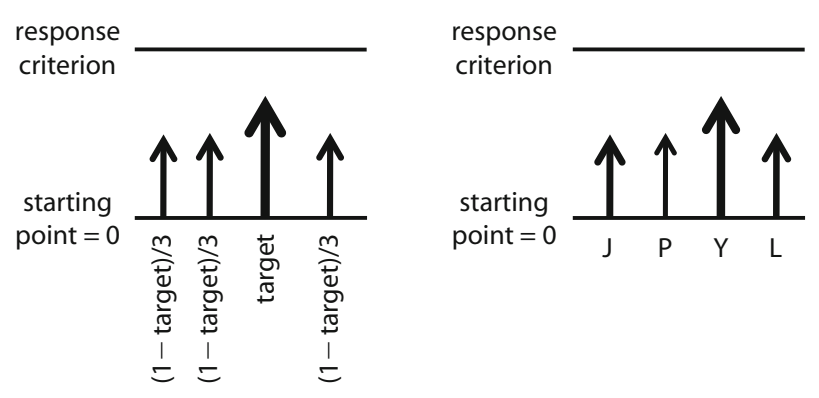

Figure 2. Arrow width represents input strength; arrow height represents amount of accumulated evidence. Left panel: Race among four accumulators driven by a single free parameter, capturing the target stimulus strength. Right panel: Race among four accumulators driven by four input strength parameters (if constrained to sum up to 1 , this race will be governed by three free parameters).

sponding to the correct alternative in each trial is modeled by one (target) input strength, and the other letter alternatives have another (foil), identical input strength (see Figure 2). Second, each letter alternative in a letter discrimination task can use a separate input strength parameter so that accumulators are assigned to specific letters. In all cases, it is assumed that the input strength parameters add to 1 (cf. Usher \& McClelland, 2001).

In addition, input strength parameters can be assumed to be the same or to differ across number of alternatives, leading to one or three sets of target-foil pairs of parameters or one or three sets of four letter-specific parameters. Input strength can also be assumed to vary across difficulty (or bias) levels, multiplying the number of input strength parameters by three. These assumptions are summarized below.

1. Three input strength parameters: one for targets in two alternatives (with the competitor's strength defined as the unit minus the target input strength); a second for targets in three alternatives (with the two competitors equally sharing the equivalent of the unit minus the target input strength); and a third for targets in four alternatives (with the three competitors equally sharing the equivalent of the unit minus the target input strength). This assumption was included to make sure that stimulus difficulty had a stronger effect on the fit measures than did the number of alternatives.

2. Nine input strength parameters: as in (1), but allowing for a different parameter in each level of difficulty (or bias) within each number of alternatives. That is, there are three target parameters among two alternatives - one for easy trials, a second for medium-difficulty trials, and a third for difficult trials - as well as three target parameters among three alternatives and three other target parameters among four alternatives.

3. Twelve input strength parameters: one set of three input strength parameters as in (1) for each of the four possible target response letters. For example, among twoalternative trials, one set of parameters modeled trials on which $\mathrm{P}$ was the target response, and another set modeled trials on which $\mathrm{L}$ was the target response.
4. Thirty-six input strength parameters: one set of nine input strength parameters as in (2) for each of the four possible target response letters, akin to (3).

\section{EXPERIMENTS 1 AND 2}

We collected data using a multiple-alternative letter discrimination task, with number of alternatives ranging from two to four across blocks. Participants saw one letter in each trial, embedded in static background noise and presented for up to $160 \mathrm{msec}$, and indicated their response by depressing the corresponding key.

We chose two manipulations: perceptual difficulty (Experiment 1) and target frequency (Experiment 2). These were chosen because we wanted to have manipulations of different cognitive processes that could lead to changes in both accuracy and RT in a relatively wide range, which, individually, could potentially be captured by a single parameter in the models. These manipulations have been successfully used to test models, and their effects have been discussed in the literature for quite some time (e.g., Hyman, 1953, and Swensson, 1972, for stimulus frequency and perceptual difficulty, respectively). In short, high-quality stimuli are expected to have an advantage over low-quality stimuli, and high-probability stimuli are expected to have an advantage over low-probability stimuli (Thomas, 2006).

Specifically, perceptual difficulty was chosen because task difficulty is also affected by the manipulation of number of alternatives. Thus, it was important to determine which factors were responsible for behavioral changes caused by an increase in task difficulty due both to the stimulus manipulation and to the manipulation of the number of alternatives. Target frequency has been used in studies of participants' bias, with bias toward one response producing increased accuracy and shorter RTs. With this manipulation, we could investigate whether the effect of adding alternatives interacts with that of bias.

\section{Method}

\section{Participants}

Five Ohio State University undergraduate students took part in the study. All the participants reported normal or corrected-to-normal vision. Each participant ran a series of 45- to 50-min sessions, for each of which they were compensated \$10. Participants 1-3 participated in both experiments; Participants 1 and 3 ran Experiment 1 (four sessions) followed by Experiment 2 (three sessions), whereas Participant 2 ran the reverse order (six sessions in Experiment 2 and five sessions in Experiment 1). Participant 4 ran only Experiment 1 (five sessions), and Participant 5 ran only Experiment 2 (six sessions).

\section{Apparatus and Procedure}

The experiments were run on personal computers running the Linux operating system with a customized real-time system. Computers were connected to a 17 -in. monitor with a resolution of $640 \times$ 480 pixels and a standard 102-key keyboard, whose numeric keypad was altered to the arrangement shown in Figure 3.

We chose P, Y, J, and L as stimulus letters on the basis of work by Gilmore, Hersh, Caramazza, and Griffin (1979). Gilmore et al. used a letter discrimination task to determine the frequency with which (capital) letters of the alphabet were confused with each other. We considered as stimulus candidates sets of four letters that were 


\section{$P_{--} Y$ 5 \\ $J_{--} \mathrm{L}$}

Figure 3. Response key arrangement on the numeric keypad of a standard keyboard. Dashed underscores represent absence of a key.

confused with one another less than $2.5 \%$ of the time, did not sound alike, and used about the same number of pixels on the computer monitor. We imposed these restrictions so that models with targetfoil input strength parameters were plausible models (see the Model Fitting section). Pilot runs with Z, H, F, and Y, for example, showed that participants were quicker to respond to $Z$ than to other letters. In subsequent debriefing, the participants reported to be first looking for the diagonal in the letter $\mathrm{Z}$, and then for the angle atop the letter $Y$ in order to make their response. If those searches failed, they reported deciding between $\mathrm{H}$ and F. No similar strategy was reported for the set P, Y, J, L.

In both experiments, a stimulus display was constructed by writing a white letter on a black background and flipping a proportion of the pixels on the screen from black to white and vice versa (e.g., Ratcliff $\&$ Rouder, 1998). We informed the participants that they would perform a simple decision task with multiple alternatives and that they were to identify an (approximately $90 \times 90$ pixel) letter appearing in a $320 \times 200$ pixel window in the center of the screen. The stimulus was displayed after the participants depressed the middle key, "5," and they used the same finger, either the index finger or the middle finger of their right hand, to press the response key corresponding to the letter. The stimulus remained on the screen for up to $160 \mathrm{msec}$. That is, if the participants released the " 5 " key in less than $160 \mathrm{msec}$, the stimulus was taken off the screen immediately after the " 5 " key release; otherwise, the stimulus was on for $160 \mathrm{msec}$. RT was measured from stimulus onset to release of the " 5 " key, and the choice of response key was also recorded. The participants received a "Too Slow" feedback message every time they took longer than $225 \mathrm{msec}$ to depress a response key after releasing the " 5 " key. In order to discourage the participants from anticipating their responses, they were given a "Too Fast" warning message for every release occurring less than $100 \mathrm{msec}$ after stimulus onset. In Experiment 2, in which anticipatory responses might occur more often, the "Too Fast" message was displayed for releases faster than 250,300 , and $350 \mathrm{msec}$ for two-, three-, and fouralternative blocks, respectively.

RT was measured from stimulus onset to the release of a key, prior to movement to any of the equally spaced response keys (cf. Figure 3) indicating the choice. This contrasts with Merkel's 10alternative design (in Hick, 1952), in which participants rested their 10 fingers on a series of 10 keys and indicated their response by depressing a key with its corresponding finger. This use of 10 keys provides no safeguard against the possibility that a participant may be able to hit keys more quickly with some fingers than with others (although the different speeds associated with different fingers may be small).

In short, the combination of very short post-key-release movements, the feedback triggered by relatively long post-key-release times, and the removal of stimulus from the display after key release (when applicable-viz., responses shorter than $160 \mathrm{msec}$ ) were safeguards in place to prevent decision processes from occurring past our recorded decision times (see G. A. Smith \& Carew, 1987, for possible participants' strategies that may distort the measurement of RT in a paradigm similar to ours). It is nevertheless possible that, in our design, some cognitive processes may still be taking place post-key-release (e.g., self-monitoring), but we know of no evidence to suggest that these processes do not occur when responses are made by keypress as well or that they are part of the decision processes we model.

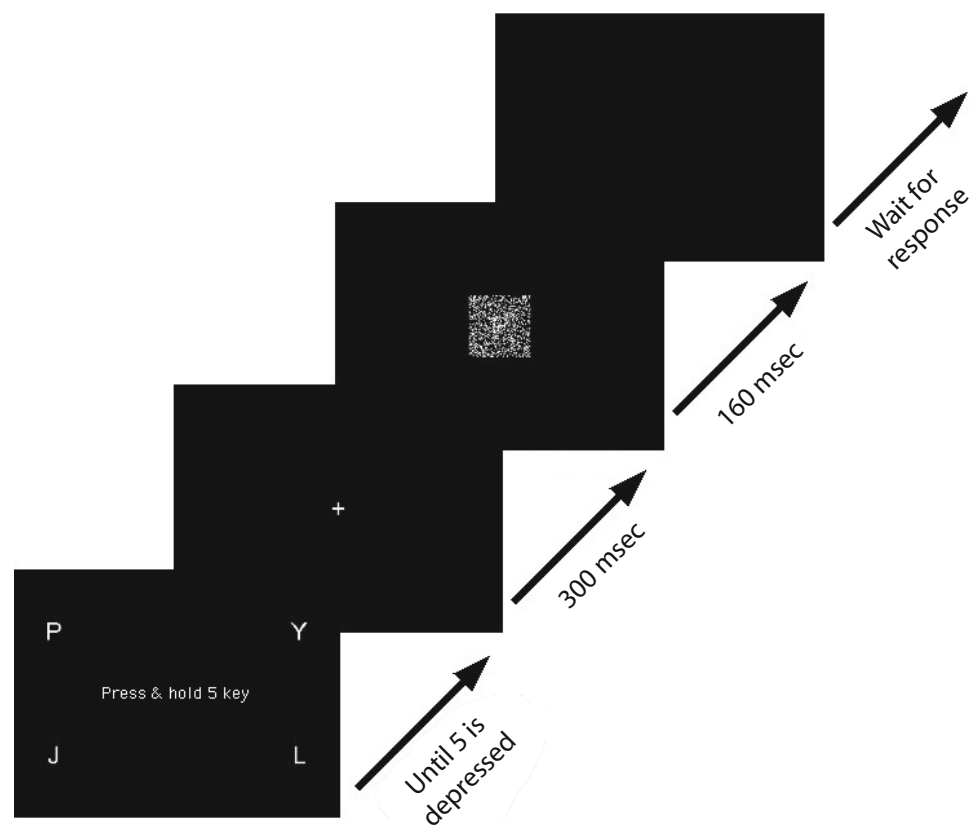

Figure 4. Trial timeline. Initial screen with instructions and the response key arrangement remained on until the " 5 " key was depressed. Then a fixation point was displayed for $\mathbf{3 0 0} \mathrm{msec}$, followed by stimulus presentation for 160 msec. A blank screen followed and remained on until the participant's response, after which the next trial began. 


\section{Theoretical Hick's Law vs. Data}

Experiment 1
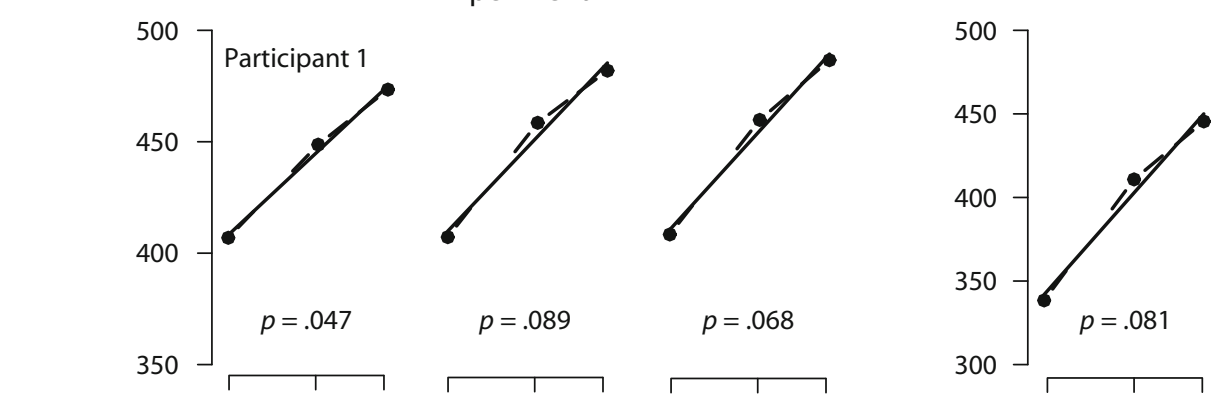

Experiment 2
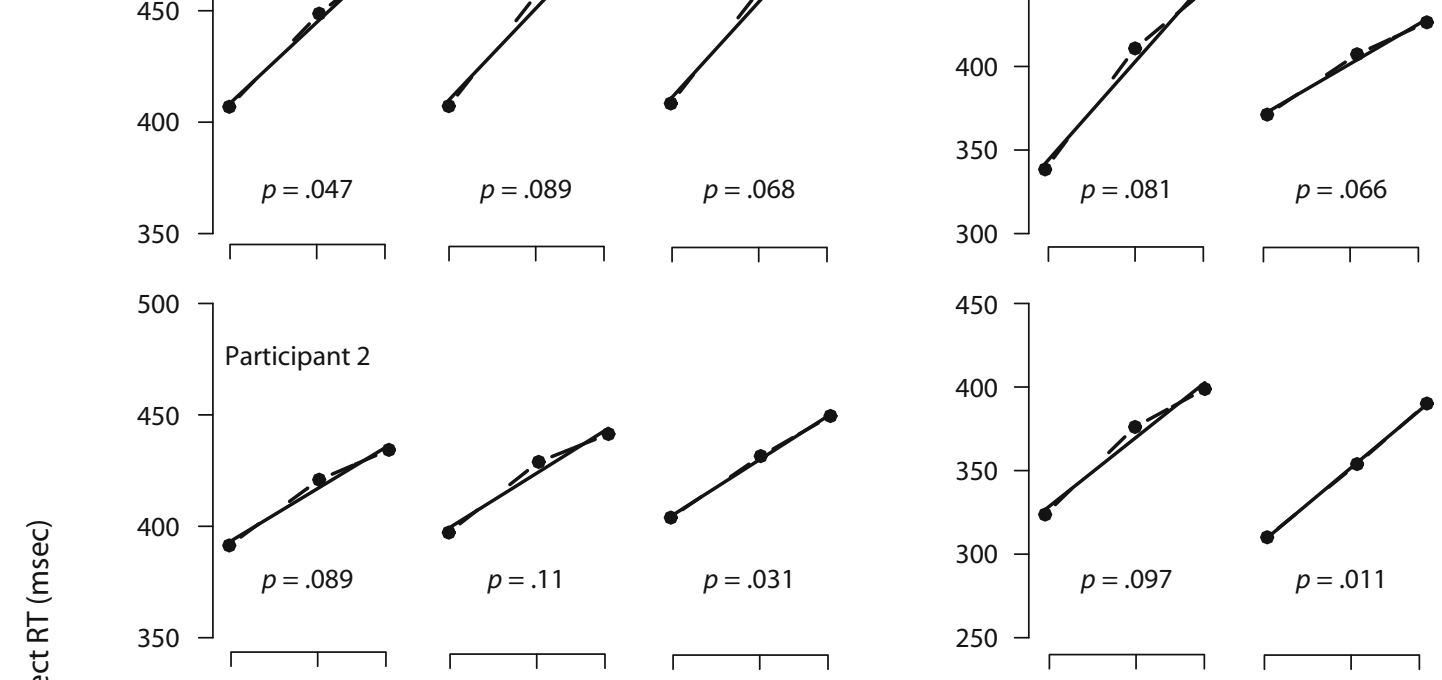

Participant 1
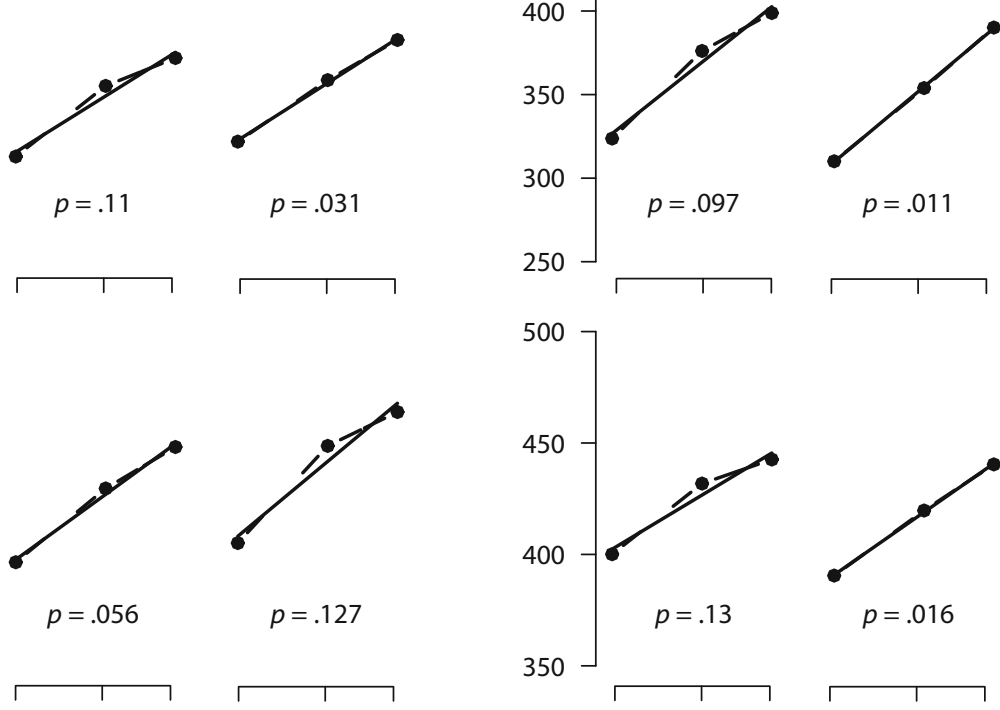

Participant 2

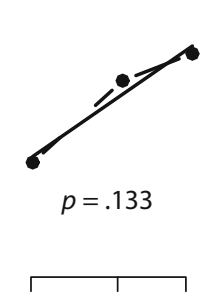

Participant 3
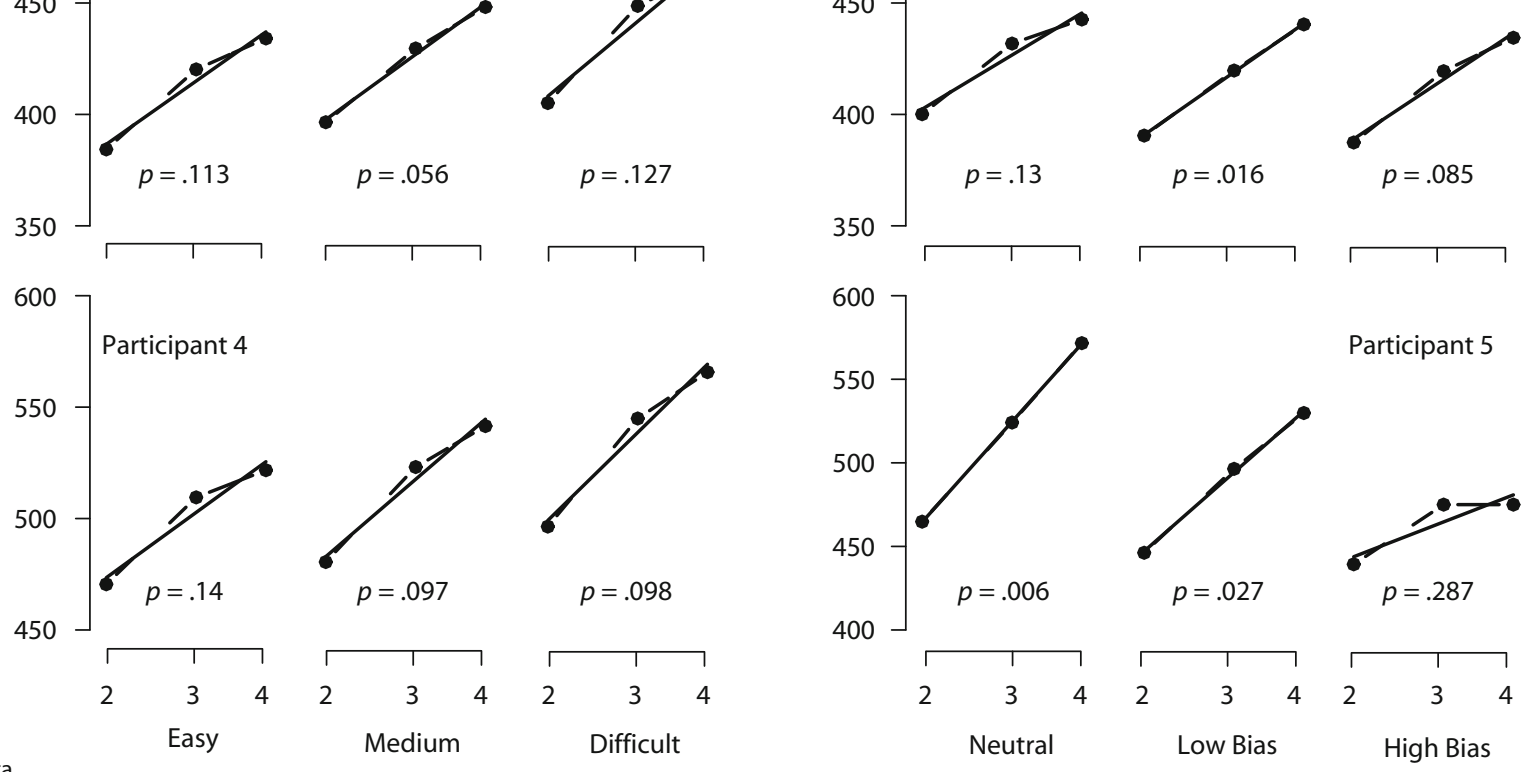

- - Data

umber of Alternatives (log)

Figure 5. Theoretical Hick's law predictions versus individual mean correct reaction times (RTs, in milliseconds) in both experiments. Note that the data in the middle and right columns in Experiment 2 (right panel) are not expected to conform to Hick's (1952) law because, by design, stimuli are not equiprobable.

Although the mapping from letter to response key did not change across trials, at the start of each trial, the response key arrangement was presented on the screen (see Figure 3), simply as a reminder for the participants. In blocks with fewer than four alternatives, a pound sign replaced unused alternatives. Figure 4 illustrates the timeline of a trial.

\section{Design}

Two $3 \times 3$ designs, detailed below, were used. In each experiment, a session was composed of 36 blocks of 60 trials each. Data from entire sessions were left out of the analyses when the participants were still familiarizing themselves with the tasks and procedures. Participants 1 and 3 previously ran some pilot sessions; hence, none 
of their sessions were excluded from our analyses. Participant 2's first two sessions in Experiment 2 and first session in Experiment 1 were excluded. Participant 4's initial session was also excluded, as well as Participant 5's initial three sessions.

Experiment 1: Difficulty. In Experiment 1, the factors were difficulty (within blocks) and number of alternatives (across blocks). In each session there were 12 two-alternative blocks, 12 three-alternative blocks, and 12 four-alternative blocks, randomly intermixed. In each block, 20 easy trials, 20 medium trials, and 20 difficult trials (i.e., respectively flipping $28.5 \%, 32.1 \%$, or $36.1 \%$ of the pixels from the white letter written on a black background from black to white and vice versa) were presented, and the participants were informed of the equal proportions of the different levels of difficulty.

Experiment 2: Proportion. In Experiment 2, the factors were stimulus proportion (across blocks) and number of alternatives (across blocks). There were three conditions in which one letter was chosen to be the high-proportion alternative (vs. the other low-proportion letters): no-, low-, and high-bias conditions. Specifically, the proportions were $30: 30,45: 15$, and $51: 9$ for the no-, low-, and high-bias conditions in two-alternative blocks; 20:20:20, $36: 12: 12$, and 45:8:7 for the no-, low-, and high-bias conditions in three-alternative blocks; and 15:15:15:15, 30:10:10:10, and 40:7:7:6 for the no-, low-, and high-bias conditions in four-alternative blocks. All the letters had an equal number of trials as the high-proportion stimulus. Stimulus difficulty was held constant: $31.6 \%$ of the pixels from the white letter written on a black background were flipped from black to white and vice versa on all the trials. In a session, there were 12 no-bias blocks, 12 low-bias blocks, and 12 high-bias blocks. Thus, among four-alternative blocks, each letter was the high-bias target once. Before each block, the participants were told how many times each alternative would appear in that block. We instructed the participants to use that information to their advantage, but not to anticipate their responses.

\section{Results}

In this section, we present the data from the two experiments. In the immediately following section, we present the model fits to the data.

\section{Number of Alternatives and Hick's Law}

Figure 5 shows mean correct RTs plotted as a function of number of alternatives averaged over Experiment 1 (left panel) and Experiment 2 (right panel). The results showed an increase in mean correct RT that is consistent with Hick's law. ${ }^{2}$ However, this relationship will not allow discrimination among the models because most will be able to predict this pattern. Slight decreases in accuracy with an increase in number of alternatives were also observed (see below), consistent with previous reports (e.g., Lacouture \& Marley, 1995).

\section{Experiment 1}

Extremely fast and slow responses were eliminated from analyses using cutoffs. The lower cutoff was chosen to be the point below which the participants performed at chance level. The upper cutoff was chosen to eliminate very slow outliers (e.g., responses $200 \mathrm{msec}$ or slower than the next fastest response) or below-chance slow responses. Averaged over participants, in Experiment 1, the mean lower cutoffs were 253,285 , and $298 \mathrm{msec}$, whereas the mean upper cutoffs were 787, 900, and $950 \mathrm{msec}$, for two, three, and four alternatives, respectively. ${ }^{3}$ Excluding data points in this way eliminated approximately $0.9 \%$ of the data. The results from Experiment 1 showed that, for all the participants, mean correct RT increased and accuracy decreased as either number of alternatives or difficulty increased (see Table 1 and Figure 6).

Plotting quantile RTs versus quantile RTs (Q-Q plots), averaged across participants, showed approximately linear relationships between conditions (cf. Ratcliff \& McKoon, 2008), whether across difficulty levels (Figure 7) or across number of alternatives (Figure 8). Linearity in Q-Q plots implies invariance in distribution shape between conditions. The closer the $\mathrm{Q}-\mathrm{Q}$ line is to the $x=y$ line, the closer to identical the distributions; a line (approximately) parallel to and above $x=y$ (i.e., slope around the unit) implies a shift of the entire distribution in the $y$ condition relative to the $x$ condition (see, e.g., Figure 7, two alternatives, error responses, medium vs. difficult).

As a function of difficulty, Q-Q lines were linear, but the slopes were greater than the unity - 1.08 , on average indicating that the increase in MRT as difficulty increased was due to RT distributions spreading (see Figure 7). Q-Q plots across number of alternatives showed shifts of the entire RT distribution (although slopes did not deviate much from the mean, 0.98), for both correct and error responses, as the number of alternatives increased (i.e., RTs became longer; see Figure 8). This shift was more prominent when number of alternatives increased from two to three than when it increased from three to four - as measured by the average difference over the five quantile RT points, approximately 45 and $23 \mathrm{msec}$ (averaged over correct and error responses), respectively. (The individual analysis does not alter the interpretation of the group data presented above and, thus, is not shown.)

Error responses were faster than correct responses for two alternatives, but not for three or four alternatives. For two alternatives, averaging across difficulty conditions, the .9-quantile point for error responses was only about $5 \mathrm{msec}$ slower than that for correct responses, whereas the .1 -quantile point was about $22 \mathrm{msec}$ faster. For three and four alternatives, there was almost no difference between error and correct responses in the .1-quantile point (about -5 and $5 \mathrm{msec}$, respectively), unlike typical two-choice results (e.g., Ratcliff \& Rouder, 2000; Thapar, Ratcliff, $\&$ McKoon, 2003), whereas error responses were slower than correct responses in the .9-quantile point (by about 22 and $29 \mathrm{msec}$, respectively).

\section{Experiment 2}

As in Experiment 1, extremely fast and slow responses were eliminated from analyses, using cutoffs. Averaged

Table 1

ANOVA for Experiment 1

\begin{tabular}{lrccl}
\hline \multicolumn{1}{c}{ Source } & $d f$ & $F$ & $\eta^{2}$ & $p$ \\
\hline Participants & 3 & $285.99^{* *}$ & .68 & .00 \\
Number of alternatives $(A)$ & 2 & $171.25^{* *}$ & .27 & .00 \\
Difficulty $(D)$ & 2 & $21.04^{* *}$ & .03 & .00 \\
$A \times D$ & 4 & 0.44 & .00 & .78 \\
Residuals & 24 & $(1,470)$ & & \\
\hline
\end{tabular}

Note-Participants is a between-subjects factor, whereas number of alternatives and difficulty are within-subjects factors. The value enclosed in parentheses represents mean squared errors. ${ }^{* *} p<.001$. 


\section{Experiment 1 Data Summary}

Accuracy
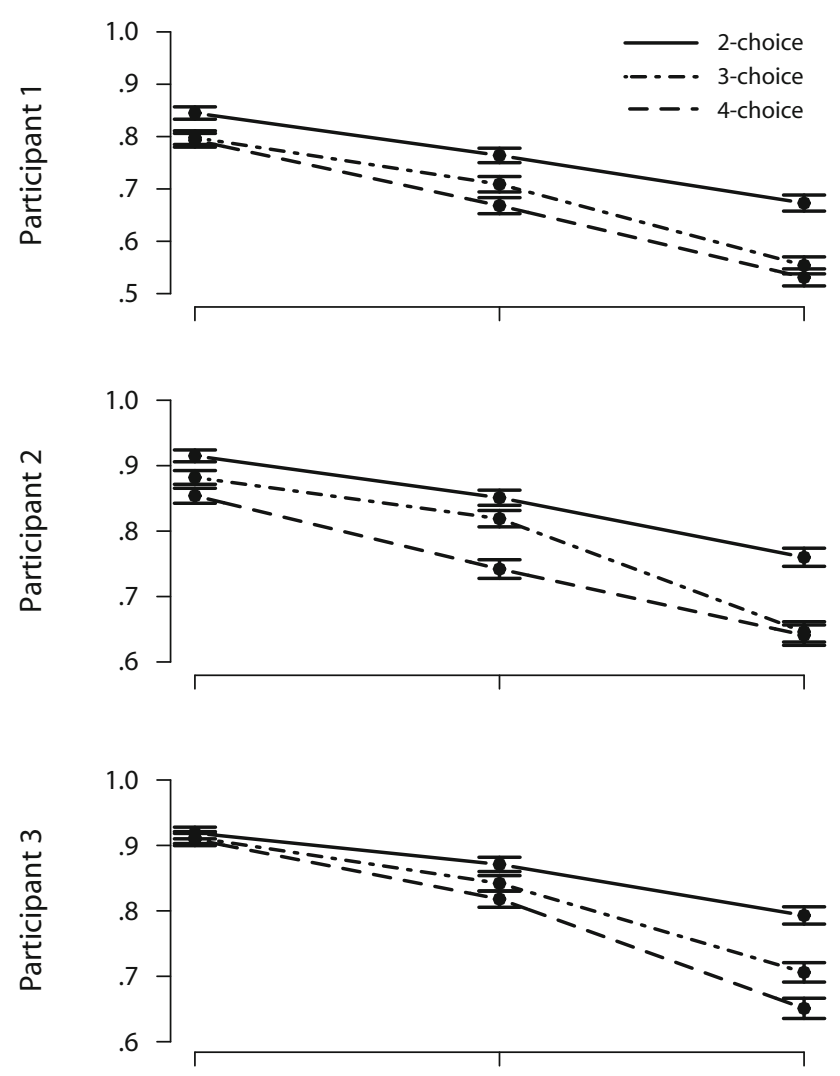
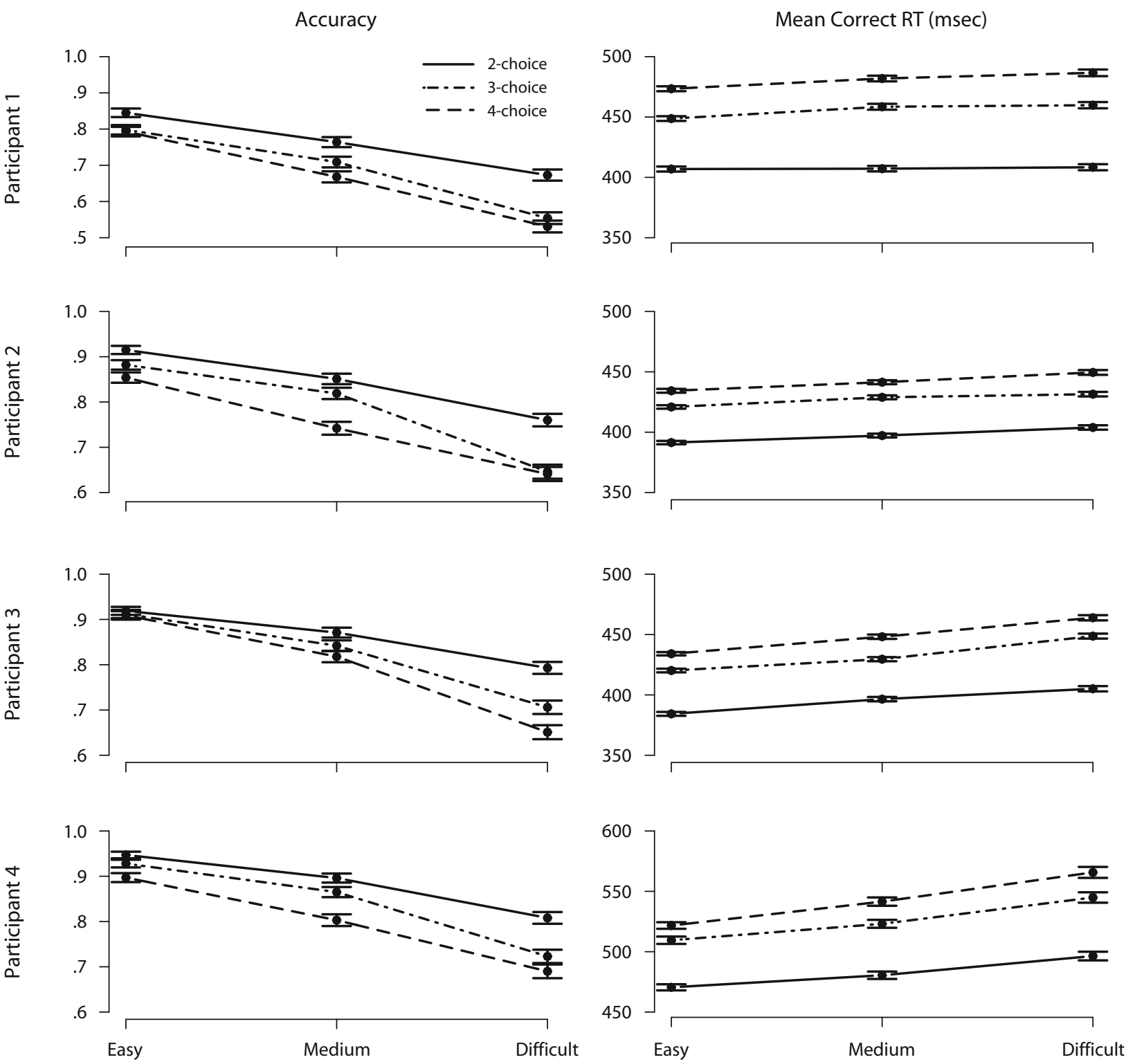

Stimulus Difficulty

Figure 6. Left panel shows that accuracy decreased with both increased number of alternatives and increased task difficulty. Right panel shows that mean correct reaction time (RT) increased with both increased number of alternatives and increased task difficulty. Error bars mark one standard error below and above each point.

over participants, in Experiment 2, the mean lower cutoffs were 213,265 , and $288 \mathrm{msec}$, whereas the mean upper cutoffs were 688,713 , and $730 \mathrm{msec}$ for two, three, and four alternatives, respectively. ${ }^{4}$ Excluding data points in this way eliminated approximately $2.8 \%$ of the data. Overall, the results from Experiment 2 showed that mean correct RTs to high-proportion stimuli decreased and accuracy increased as blocks became more biased. Also as in Experiment 1, mean correct RT increased and accuracy decreased as number of alternatives increased (see Table 2 and Figure 9).
The Q-Q plots for Experiment 2 (Figure 10) were linear, as in Experiment 1, and showed little change in the shape of RT distributions as bias level increased (for both correct and error responses). They also showed a larger shift of the entire correct RT distribution when bias increased from low to high than when bias increased from none to low (approximately 15 and $8 \mathrm{msec}$, respectively, averaged across number of alternatives). As in Experiment 1, fast error responses were obtained for two alternatives, but not for three or four alternatives. 


\section{Across-Difficulty Q-Q Plots: Experiment 1}

Averaged Quantile RTs (msec)

Correct Responses

Error Responses
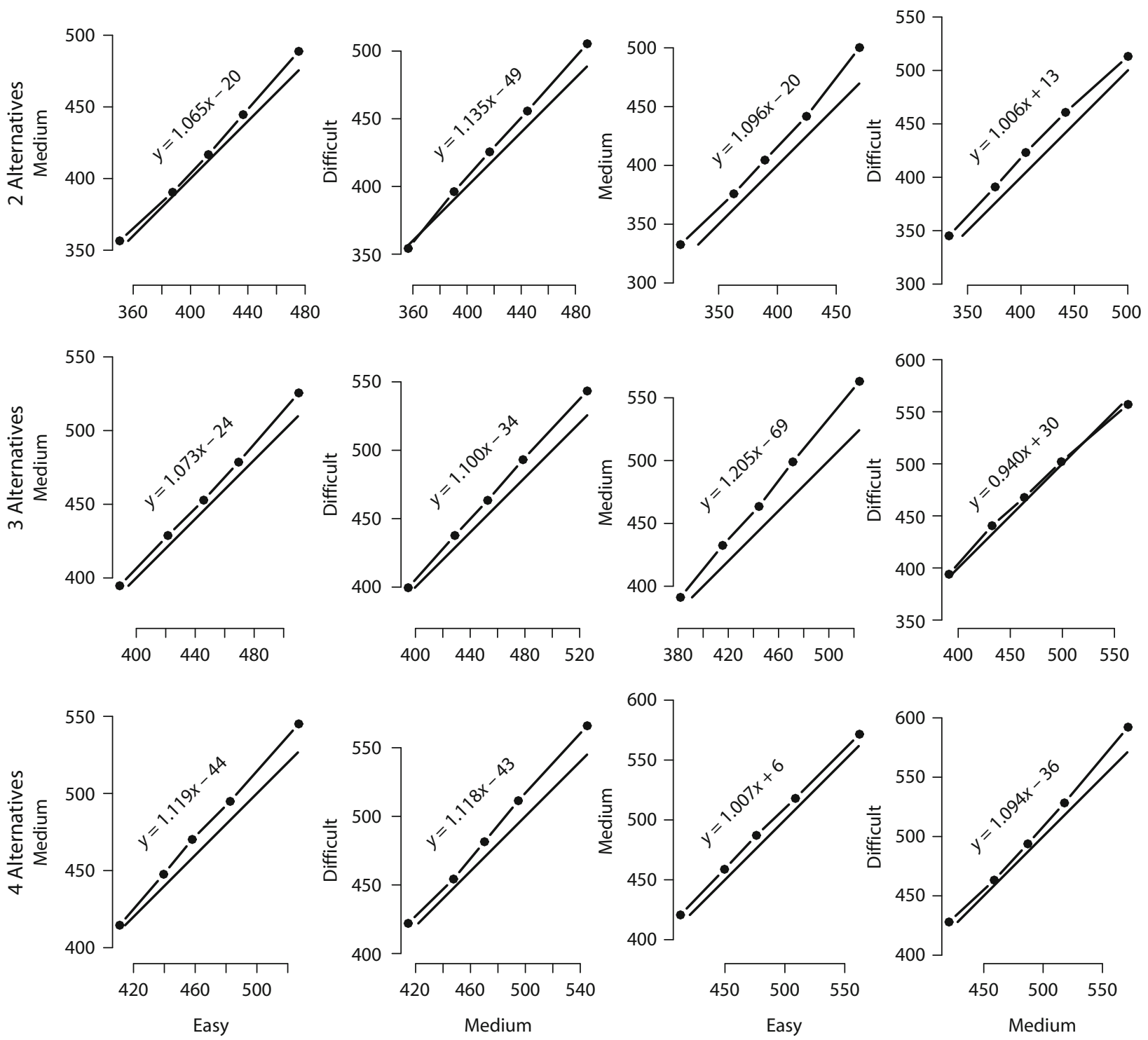

Figure 7. Quantile-quantile (Q-Q) plots drawn from averaging the individual quantiles computed for each participant in Experiment 1 (represented by the dots connected by lines). The (dotless) continuous solid lines represent $x=y$. Equation $y=a x+b(a=$ slope; $b=$ intercept) is the regression line of $y$ on $x$; slopes near the unit indicate nearly identical distributions between $y$ and $x$, slopes greater than the unit show spreading of the tail in $y$ versus $x$, and slopes below the unit show spreading of the leading edge of the $y$ distribution in comparison with $x$. Plots show very little difference in the shape of reaction time (RT) distributions as a function of difficulty, for all numbers of alternatives.

Q-Q plots of no bias versus low bias and low bias versus high bias (Figure 11) showed a shift of the entire RT distribution, for both correct and error responses, as the number of alternatives increased and RTs became longer. This shift was less prominent when number of alternatives increased from three to four than when it increased from two to three (respectively, approximately 26 and $55 \mathrm{msec}$, averaged over correct and error responses). Regardless of the magnitude of the shift in RT distributions, there was little change in their shapes. In short, the increase of both bias and number of alternatives simply shifted the RT distributions.

\section{MODELING ANALYSIS}

There were 384 possible models from the combinations of the characteristics we presented earlier. To reduce this number to a more manageable number, we took two steps: 


\section{Across-Alternatives Q-Q Plots: Experiment 1}

Averaged Quantile RTs (msec)

Correct Responses

Error Responses
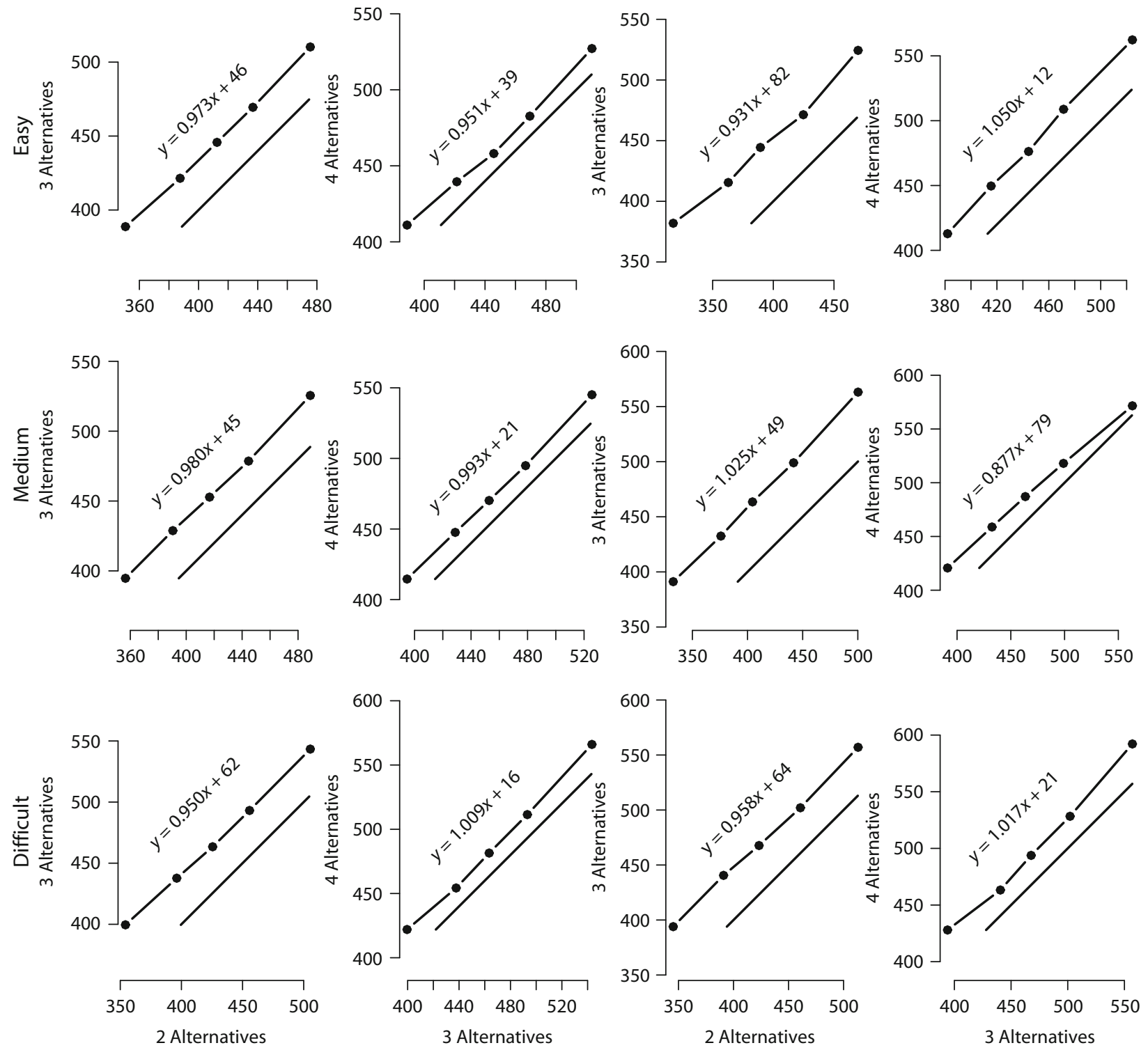

Figure 8. Quantile-quantile (Q-Q) plots drawn from averaging the individual quantiles computed for each participant in Experiment 1 (represented by the dots connected by lines). The (dotless) continuous solid lines represent $x=y$. Equation $y=a x+b(a=$ slope; $b=$ intercept) is the regression line of $y$ on $x$; slopes near the unit indicate nearly identical distributions between $y$ and $x$, slopes greater than the unit show spreading of the tail in $y$ versus $x$, and slopes below the unit show spreading of the leading edge of the $y$ distribution in comparison with $x$. Plots show shift in both correct and error reaction time (RT) distributions across difficulty levels. As number of alternatives increased, RTs became longer. Very little difference in RT distribution shape was observed.

First, by simulation, we evaluated the impact of each model parameter on the model's predictions (even though these simulations are susceptible to parameter interaction effects); and second, we performed preliminary tests with the data from Experiment 1. On the basis of the first step, we found the following.

1. Decay. As decay increases, accuracy increases and spread of RT distributions increases, accompanied by slight increase in leading edge, and these changes are stronger for larger values of decay.

2. Inhibition. Small increases in inhibition cause an increase in accuracy and an increase in spread of the RT distribution with negligible changes in the leading edge of RT distributions. When inhibition was high, change in the spread of the RT distribution was small for two-choice conditions but larger for three- and four-choice conditions. 
Table 2

ANOVA for Experiment 2

\begin{tabular}{lrccl}
\hline \multicolumn{1}{c}{ Source } & $d f$ & $F$ & $\eta^{2}$ & $p$ \\
\hline Participants & 3 & $231.83^{* *}$ & .73 & .00 \\
Number of alternatives $(A)$ & 2 & $108.24^{* *}$ & .23 & .00 \\
Proportion of stimuli $(P)$ & 2 & $8.71^{*}$ & .02 & .00 \\
$A \times P$ & 4 & 1.16 & .00 & .35 \\
Residuals & 24 & $(145)$ & & \\
\hline
\end{tabular}

Note-Participants is a between-subjects factor, whereas number of alternatives and proportion of stimuli are within-subjects factors. The value enclosed in parentheses represents mean squared errors. ${ }^{*} p<$ $.01 . \quad * * p<.001$

3. Decay and inhibition. When decay and inhibition have the same nonzero value (i.e., are balanced), almost identical leading edges are observed in the RT distributions, when compared with zero values of decay and inhibition. Nonzero values of decay and inhibition also lead to RT distributions with slightly larger spread than those obtained with zero values of decay and inhibition.

4. Starting point. Adding relatively small amounts of variability to starting points (i.e., one eighth or one fourth of the range from 0 to threshold) led to slight leftward shifts of the RT distributions. For negatively correlated starting points, a slight decrease in accuracy was observed, whereas no clear pattern was observed for random starting points.

5. Negative evidence versus nonnegative-only evidence. Negatively unbounded accumulation of evidence led to an increase in accuracy and a slight increase in the spread of the RT distributions, when compared with nonnegativeonly accumulation.

6. Number of nondecisional components. When nondecisional components are ordered such that nondecisional time for two alternatives is shorter than that for three alternatives, which in turn is shorter than that for four alternatives, reducing the number of nondecisional components from three to one (estimated in between the time for two and three alternatives) caused a rightward shift in the twochoice RT distributions and a leftward shift in the threeand four-choice distributions.

7. Decision criterion. By itself, increasing the number of decision criteria from one (identical criteria for two, three, and four alternatives) to three (different criteria for two, three, and four alternatives) causes changes in both accuracy and RT distributions that are not readily interpretable unless the three criteria are ordered such that the criterion threshold for two alternatives is lower than that for three alternatives, which in turn is lower than that for four alternatives. When that holds, accuracy will decrease, and RT distributions will have a slightly larger spread as number of alternatives increases.

8. Input strength. Having 3 or 12 input strength parameters with "average" values did not allow the models to capture changes in difficulty conditions. The advantage of having 12 parameters - the preferred structure to fit our data-over 3 was the ability to model the accumulation of each letter alternative individually. Having 36 input strength parameters also allowed small changes in RT distributions and accuracy to be modeled as a function of specific letters, but such models' Bayesian information cri- terion (BIC) values were much higher than the BIC values for alternative models, due to the number of parameters.

9. Decision criterion, input strength, and decay. We also note that increasing the value of the decision criterion, reducing the input strength value, or increasing decay leads to similar slowing of RT distributions.

We were able to rule out several models because neither did they qualitatively fit the data from Experiment 1 nor were their BIC values competitive.

1. Models with inhibition. We found that the extra parameter representing mutual lateral inhibition worsened the BIC estimates without producing qualitatively better fits, relative to the models that did not have such inhibition.

2. Models with noncorrelated, nonidentical starting points. We found that models with identical or negatively correlated starting points produced competitive BIC estimates, but models with random starting points produced higher BIC estimates.

3. Models that allowed negative evidence. These models produced poorer qualitative fits than did the models with nonnegative evidence only.

4. Models with 3 (target/foil, across number of alternatives) or 12 (one for each of the four letters, across number of alternatives) input strength parameters. These models assumed that the experimental difficulty manipulations would be absorbed by parameters other than the input strength parameters, and, hence, they were ruled out on both theoretical and empirical grounds.

5. Models with 36 (one for each of the four letters, across number of alternatives and difficulty conditions) input strength parameters. These variants did not produce competitive BIC estimates, due to the much larger number of parameters than for the models with nine input strength parameters (target/foil, across number of alternatives and difficulty conditions). Because our choice of letter alternatives was designed to use a set of letters that produced about the same confusability (cf. Gilmore et al., 1979), the exclusion of these models (which modeled accumulation rates for each specific letter separately) does not result in a loss of generality.

Excluding these least competitive classes of models reduced the number of model variants to 16 , involving the following differences: three versus one nondecisional component, three versus one decision criterion, decaying versus nondecaying accumulation, and identical versus different starting points (we used negatively correlated starting points for the data in Experiment 1 and biased starting points for the data in Experiment 2; in the latter case, target starting points will be closer to criterion threshold than will the starting points of competing accumulators in the same proportion as the response alternative bias). Table 3 lists the structure of all the models tested. Note that all the LA variants use noncompeting racing processes with decay, which are dual diffusion models, as in Ratcliff et al. (2007).

\section{Model Fitting}

We pooled each participant's data across sessions, provided that the session-by-session data showed relatively stable performance, as measured by MRT and accuracy. 


\section{Experiment 2 Data Summary}

Accuracy
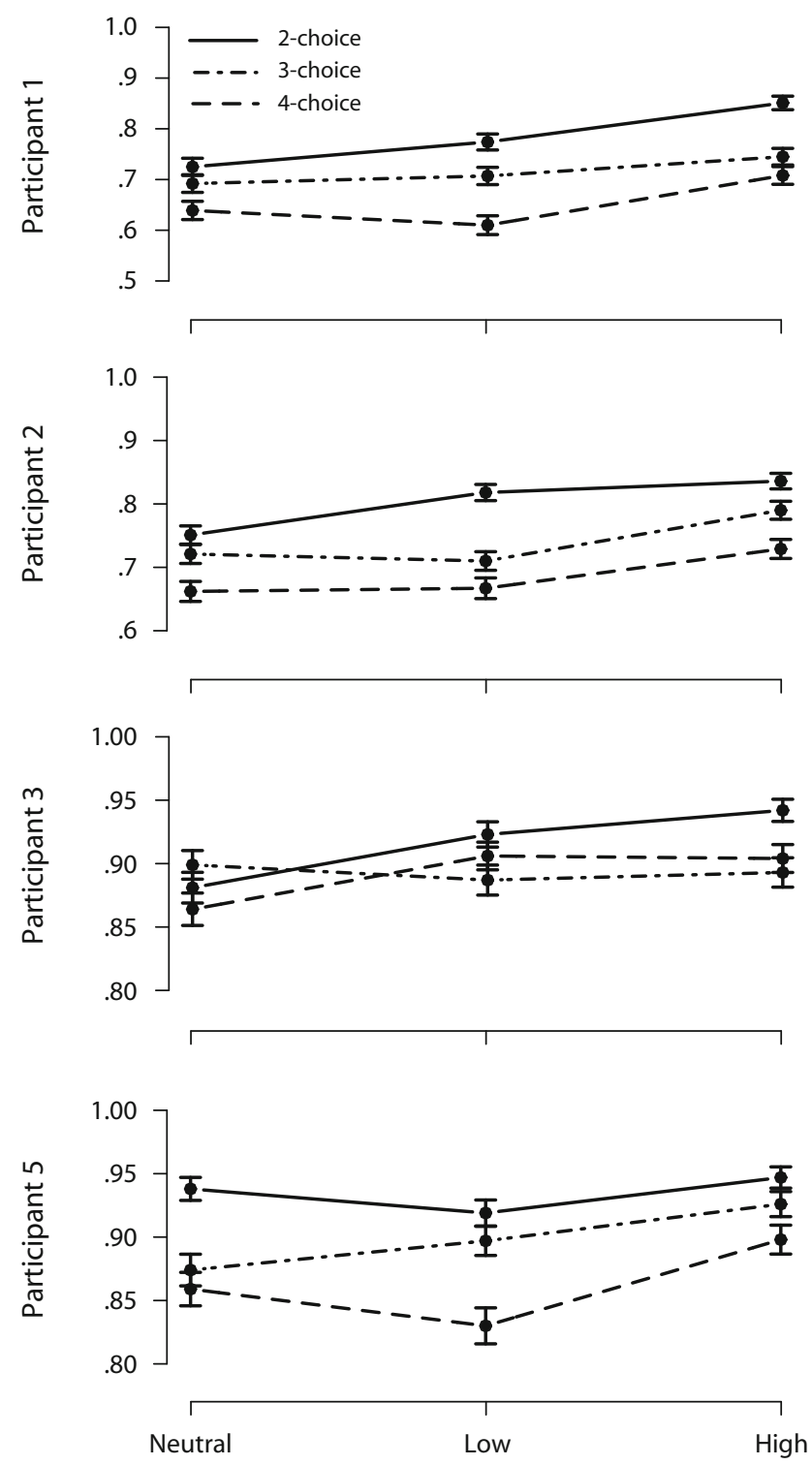

Mean Correct RT (msec)
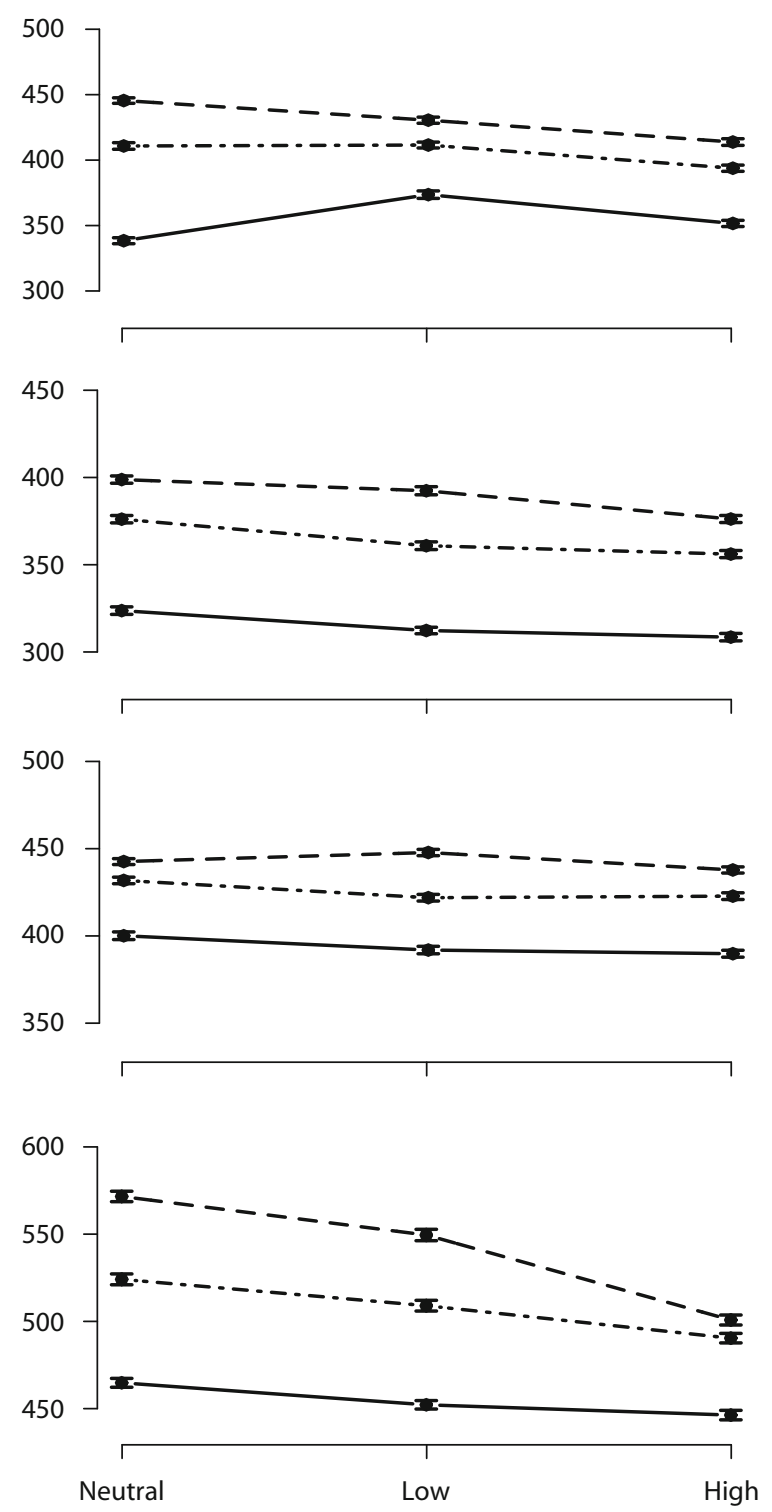

Bias Condition

Figure 9. Left panel shows that accuracy decreased with an increase in the number of alternatives (except for Participant 3) and increased from low- to high-bias conditions. The right panel shows that mean correct reaction time (RT) increased with an increase in the number of alternatives and decreased from low- to high-bias conditions. Error bars mark one standard error below and above each point.

Specifically, the data from initial sessions up to session $i$ were removed from the analyses if the data from session $i+1$ showed that responses were faster by more than $10 \%$ on average or more accurate by more than 0.1 percentage point in any condition.

RT data were separated into error and correct RTs, and error and correct RT distributions were approximated by five quantiles, evenly spaced between .1 and .9. Each model was simultaneously fit to error and correct RT distributions from each individual participant. Goodness-of- fit measures were computed for each model and for each participant.

As in the preliminary tests, the main statistic we used in the minimization routines to adjust the models' parameters in search of the best fit was BIC. We checked parameter estimates obtained from minimization with BIC against parameter estimates obtained from minimization with chi-square $\left(\chi^{2}\right)$ and verified that these estimates were consistent. Hence, interpretation of parameter estimates of any model was not BIC specific. We used BIC to evalu- 


\section{Across-Bias Q-Q Plots: Experiment 2}

\section{Averaged Quantile RTs (msec)}

Correct Responses

Error Responses
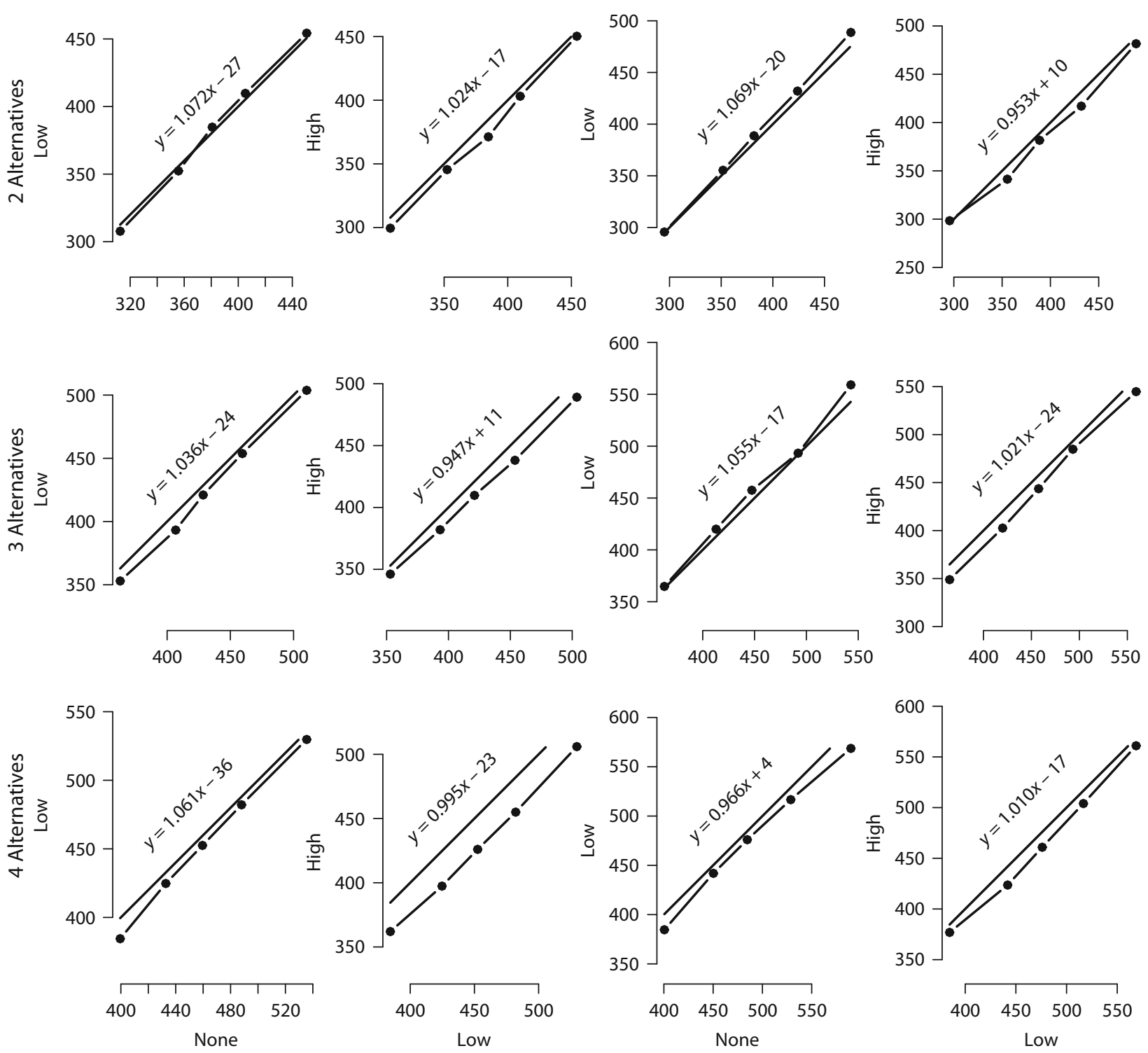

Figure 10. Quantile-quantile $(Q-Q)$ plots drawn from averaging the individual quantiles computed for each participant in Experiment 2 (represented by the dots connected by lines). The (dotless) continuous solid lines represent $x=y$. Equation $y=a x+b(a=\operatorname{slope}$; $b=$ intercept) is the regression line of $y$ on $x$; slopes near the unit indicate nearly identical distributions between $y$ and $x$, slopes greater than the unit show spreading of the tail in $y$ versus $x$, and slopes below the unit show spreading of the leading edge of the $y$ distribution in comparison with $x$. Nearly identical reaction time (RT) distributions were observed across bias conditions for two alternatives, but faster correct RT distributions were observed in the high-bias condition than in the low-bias condition for four alternatives.

ate how well the models fit the data because we needed to compare nonnested models with different numbers of parameters, a situation for which $\chi^{2}$ ranking is inadequate. In addition, comparing models using their BIC estimates can be done in a statistically meaningful and simple way, as described by Raftery (1995).

BIC provides a penalty for the number of parameters in a model, and it penalizes models for complexity of their functional form (cf. Schwarz, 1978; Wasserman, 2000).
Since our minimization routine was based on five quantile RTs for correct responses and five for error responses, creating six data bins for each case, the BIC statistic is defined by

$$
\mathrm{BIC}=-2\left[\sum_{i} N p_{i} \ln \left(\pi_{i}\right)\right]+M \ln (N),
$$

where $p_{i}$ and $\pi_{i}$ are the proportions of observed and predicted data in the $i$ th bin, $N$ is the number of observations 


\section{Across-Alternatives Q-Q Plots: Experiment 2}

\section{Averaged Quantile RTs (msec)}

Correct Responses

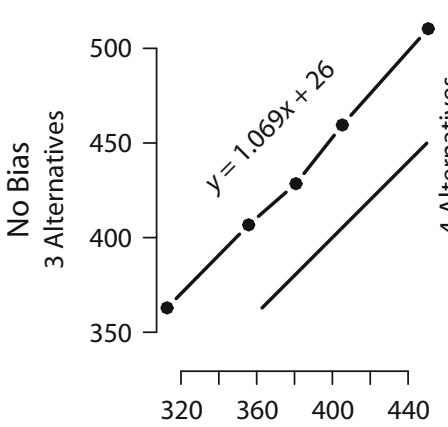

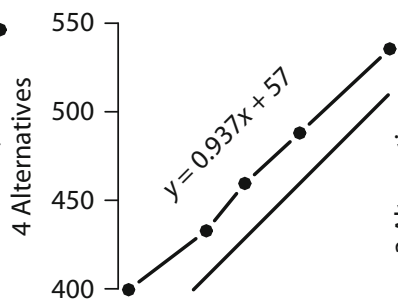

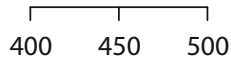

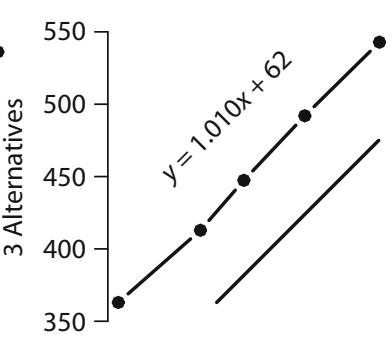

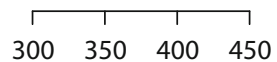

Error Responses
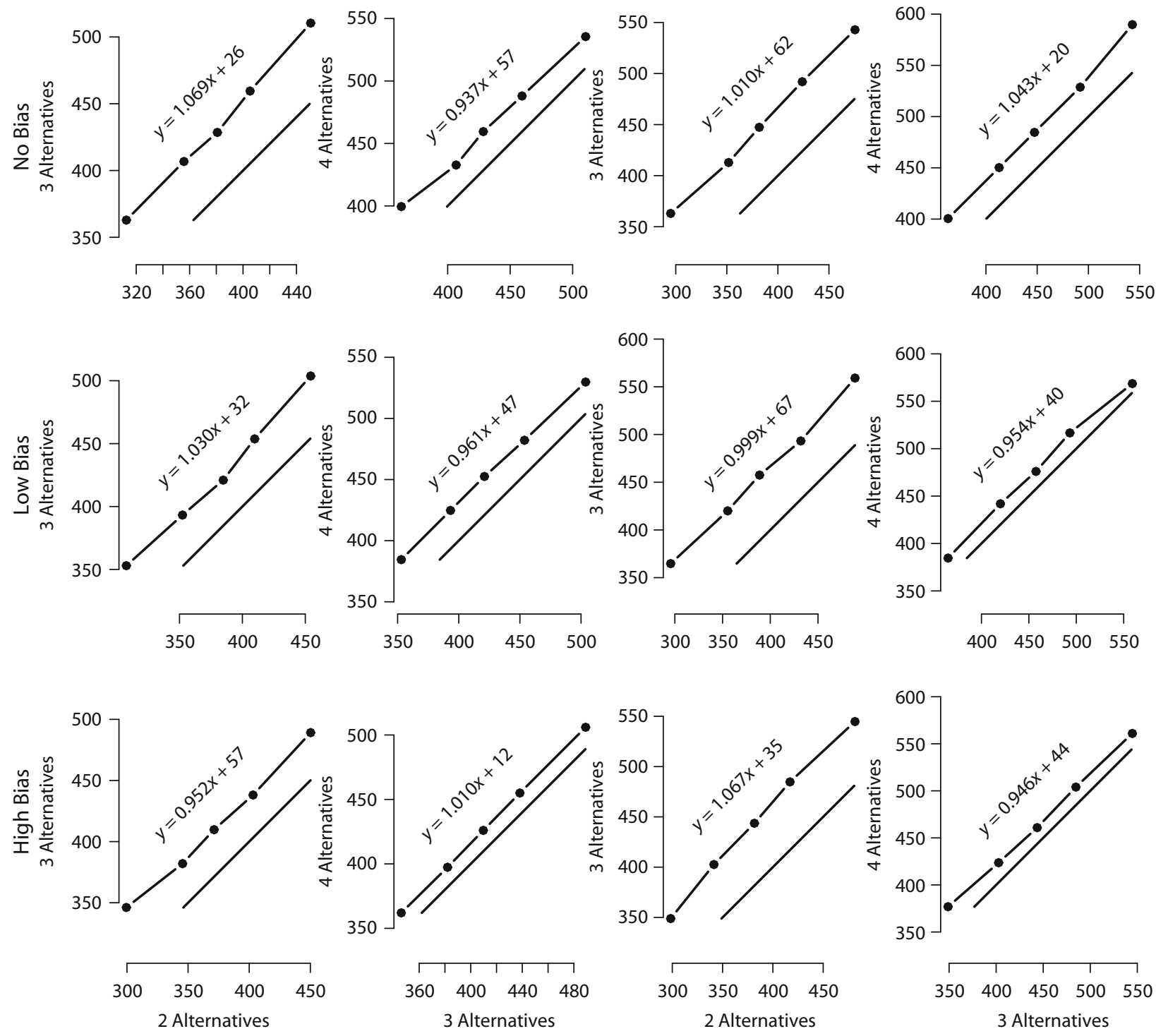

Figure 11. Quantile-quantile (Q-Q) plots drawn from averaging the individual quantiles computed for each participant in Experiment 2 (represented by the dots connected by lines). The (dotless) continuous solid lines represent $x=y$. Equation $y=a x+b(a=$ slope; $b=$ intercept) is the regression line of $y$ on $x$; slopes near the unit indicate nearly identical distributions between $y$ and $x$, slopes greater than the unit show spreading of the tail in $y$ versus $x$, and slopes below the unit show spreading of the leading edge of the $y$ distribution in comparison with $x$. Dots above $x=y$ show longer reaction times (RTs) for a higher number of alternatives.

in the condition, and $M$ is the number of free parameters in the model.

The models were fit to data using the SIMPLEX fitting method (Nelder \& Mead, 1965). Because there is no known explicit solution for Equation 3, predictions from the models were obtained by simulation. We used Monte Carlo methods that generated 20,000 simulations of the decision process to compute the probabilities of the responses and their respective RT distributions. In the fitting method, each 20,000 simulations represented one iteration. The first iteration was based on a set of starting parameters, and the iterations that followed were based on the SIMPLEX adjustments to that set of starting parameters. The last iteration in a (fitting) run produced an optimal set of parameters, based on the minimum BIC (or $\chi^{2}$ ) value obtained. In an attempt to avoid local minima, we used three starting-parameter sets, each of which was used to start a different serial chain of four SIMPLEX fits (in which each optimal set of parameters produced by a run was used as the starting set of parameters for 
Table 3

Structure of Models Tested

\begin{tabular}{lcccccc}
\hline & \multicolumn{2}{c}{ Constraint } & & & \multicolumn{2}{c}{ Parameter } \\
\cline { 2 - 3 } \cline { 6 - 7 } \multicolumn{1}{c}{ Model } & Evidence & St. Pt. & Decay & Inh. & $t_{\text {er }}$ & Criterion \\
\hline $\mathrm{A}(1 T, 1 C, e S)$ & $\geq 0$ & $=$ & no & no & 1 & 1 \\
$\mathrm{~A}(1 T, 1 C, c S \mid b S)$ & $\geq 0$ & cor & no & no & 1 & 1 \\
$\mathrm{~A}(1 T, 3 C, e S)$ & $\geq 0$ & $=$ & no & no & 1 & 3 \\
$\mathrm{~A}(1 T, 3 C, c S \mid b S)$ & $\geq 0$ & cor & no & no & 1 & 3 \\
$\mathrm{~A}(3 T, 1 C, e S)$ & $\geq 0$ & $=$ & no & no & 3 & 1 \\
$\mathrm{~A}(3 T, 1 C, c S \mid b S)$ & $\geq 0$ & cor & no & no & 3 & 1 \\
$\mathrm{~A}(3 T, 3 C, e S)$ & $\geq 0$ & $=$ & no & no & 3 & 3 \\
$\mathrm{~A}(3 T, 3 C, c S \mid b S)$ & $\geq 0$ & cor & no & no & 3 & 3 \\
\hline
\end{tabular}

Note-The eight models without decay shown here are structurally analogous to the eight models with decay (not shown). The other eight models tested are identified by LA, meaning leaky accumulator (with decay), otherwise with the same structures. St. Pt., starting point; Inh., inhibition; $t_{\mathrm{er}}$, nondecision time; cor, negatively correlated. Model variant labels abbreviate the models' structure: A, accumulator (without decay); $T, t_{\mathrm{er}} ; C$, criterion; $c S$, correlated starting point (Experiment 1 only); $b S$, biased starting point (Experiment 2 only); $e S$, equal starting point.

Table 4

Bayesian Information Criterion Rankings (BIC:Rk) for Experiment 1

\begin{tabular}{lllll}
\hline & \multicolumn{4}{c}{ BIC:Rk } \\
\cline { 2 - 5 } \multicolumn{1}{c}{ Model } & Participant 1 & Participant 2 & Participant 3 & Participant 4 \\
\hline $\mathrm{A}(1 T, 1 C, e S)$ & $42,311: 14$ & $41,272: 14$ & $40,574: 14$ & $37,277: 2$ \\
$\mathrm{~A}(1 T, 1 C, c S)$ & $41,133: 11$ & $40,772: 13$ & $40,011: 11$ & $37,401: 6$ \\
$\mathrm{~A}(1 T, 3 C, e S)$ & $40,938: 6$ & $40,150: 4$ & $39,684: 5$ & $37,535: 12$ \\
$\mathrm{~A}(1 T, 3 C, c S)$ & $41,022: 8$ & $40,252: 7$ & $39,776: 7$ & $37,523: 11$ \\
$\mathrm{~A}(3 T, 1 C, e S)$ & $40,669: 1$ & $39,945: 1$ & $39,420: 1$ & $37,304: 4$ \\
$\mathrm{~A}(3 T, 1 C, c S)$ & $40,772: 2$ & $40,042: 2$ & $39,530: 2$ & $37,417: 7$ \\
$\mathrm{~A}(3 T, 3 C, e S)$ & $40,887: 4$ & $40,165: 5$ & $39,689: 5$ & $37,397: 5$ \\
$\mathrm{~A}(3 T, 3 C, c S)$ & $41,165: 12$ & $40,472: 11$ & $40,155: 13$ & $37,725: 16$ \\
$\mathrm{LA}(1 T, 1 C, e S)$ & $42,958: 16$ & $41,609: 16$ & $40,838: 15$ & $37,694: 15$ \\
$\mathrm{LA}(1 T, 1 C, c S)$ & $42,618: 15$ & $41,424: 15$ & $40,899: 16$ & $37,675: 14$ \\
$\mathrm{LA}(1 T, 3 C, e S)$ & $41,110: 9$ & $40,273: 8$ & $39,820: 9$ & $37,453: 9$ \\
$\mathrm{LA}(1 T, 3 C, c S)$ & $41,228: 13$ & $40,478: 11$ & $40,034: 12$ & $37,578: 13$ \\
$\mathrm{LA}(3 T, 1 C, e S)$ & $40,784: 3$ & $40,060: 3$ & $39,557: 3$ & $37,248: 1$ \\
$\mathrm{LA}(3 T, 1 C, c S)$ & $40,877: 4$ & $40,175: 5$ & $39,655: 4$ & $37,291: 3$ \\
$\mathrm{LA}(3 T, 3 C, e S)$ & $41,007: 7$ & $40,297: 9$ & $39,775: 7$ & $37,435: 8$ \\
$\mathrm{LA}(3 T, 3 C, c S)$ & $41,102: 9$ & $40,416: 10$ & $39,881: 10$ & $37,509: 10$ \\
\hline
\end{tabular}

Note-Ties indicate that one model did not have very strong support over the other (i.e., $p>.99$; see Raftery, 1995, Table 6). Model variant labels abbreviate the models' structure: A, accumulator (without decay); LA, leaky accumulator (with decay); $T, t_{\mathrm{er}} ; C$, criterion; $C S$, correlated starting point; $e S$, equal starting point.

Table 5

Bayesian Information Criterion (BIC) Versus Akaike Information Criterion (AIC) Rankings (Rk) of the Six Best-Fitting Models in Experiment 1

\begin{tabular}{|c|c|c|c|c|c|c|c|c|}
\hline \multirow[b]{2}{*}{ Model } & \multicolumn{2}{|c|}{ Participant 1} & \multicolumn{2}{|c|}{ Participant 2} & \multicolumn{2}{|c|}{ Participant 3} & \multicolumn{2}{|c|}{ Participant 4} \\
\hline & BIC:Rk & AIC:Rk & BIC:Rk & AIC:Rk & BIC:Rk & AIC:Rk & BIC:Rk & AIC:Rk \\
\hline $\mathrm{A}(1 T, 3 C, e S)$ & $40,938: 4$ & $39,591: 5$ & $40,150: 3$ & $38,832: 5$ & $39,684: 3$ & $38,386: 5$ & $37,535: 6$ & $36,266: 6$ \\
\hline $\mathrm{A}(3 T, 1 C, e S)$ & $40,669: 1$ & $39,322: 1$ & $39,945: 1$ & $38,627: 1$ & $39,420: 1$ & $38,121: 1$ & $37,304: 2$ & $35,896: 2$ \\
\hline $\mathrm{A}(3 T, 3 C, e S)$ & $40,887: 3$ & $39,360: 3$ & $40,165: 4$ & $38,671: 3$ & $39,689: 3$ & $38,218: 3$ & $37,397: 3$ & $35,959: 4$ \\
\hline $\mathrm{LA}(1 T, 3 C, e S)$ & 41,110:6 & $39,674: 6$ & $40,273: 5$ & $38,867: 6$ & $39,820: 6$ & $38,435: 6$ & $37,453: 5$ & $36,100: 5$ \\
\hline $\mathrm{LA}(3 T, 1 C, e S)$ & $40,784: 2$ & $39,347: 2$ & $40,110: 2$ & $38,654: 2$ & $39,557: 2$ & $38,173: 2$ & $37,248: 1$ & $35,895: 1$ \\
\hline $\mathrm{LA}(3 T, 3 C, e S)$ & $41,007: 5$ & $39,391: 4$ & $40,323: 6$ & $38,716: 4$ & $39,775: 5$ & $38,218: 4$ & $37,435: 4$ & $35,914: 3$ \\
\hline
\end{tabular}

Note-A tie (in BIC rankings) indicates that one model did not have very strong support over the other (i.e., $p>.99$; see Raftery, 1995, Table 6). Model variant labels abbreviate the models' structure: A, accumulator (without decay); LA, leaky accumulator (with decay); $T, t_{\mathrm{er}} ; C$, criterion; $e S$, equal starting point. 
Table 6

Mean Parameter Estimates for the Three Best-Fitting Models in Experiment 1

\begin{tabular}{|c|c|c|c|c|c|c|c|c|c|c|c|c|c|c|c|c|c|c|}
\hline Model & $T_{\mathrm{er}}^{2}$ & $T_{\mathrm{er}}^{3}$ & $T_{\mathrm{er}}^{4}$ & $s_{t}$ & & $c^{2}$ & $c^{3}$ & $c^{4}$ & $\sigma$ & $\rho_{\mathrm{e}}^{2}$ & $\rho_{\mathrm{m}}^{2}$ & $\rho_{\mathrm{d}}^{2}$ & $\rho_{\mathrm{e}}^{3}$ & $\rho_{\mathrm{m}}^{3}$ & $\rho_{\mathrm{d}}^{3}$ & $\rho_{\mathrm{e}}^{4}$ & $\rho_{\mathrm{m}}^{4}$ & $\rho_{\mathrm{d}}^{4}$ \\
\hline & & & & & 0 & & & & & & & & & & & & & \\
\hline & & & & & & & & & & & & & & & & & & \\
\hline$\left(3 T, 3 C, e_{i}\right.$ & .325 & 354 & 364 & .106 & 0 & 0.815 & 0.899 & 0.980 & 0.455 & .917 & .796 & .689 & .922 & .782 & .604 & .926 & .765 & .59 \\
\hline
\end{tabular}

Note-Average of the parameter estimates across all 4 participants. $T_{\mathrm{er}}^{n}$, nondecision time (in seconds) for the corresponding $n$ number of alternatives; $s_{t}$, range of variability in $t_{\mathrm{er}} ; c^{n}$, criterion for the corresponding $n$ number of alternatives; $\sigma, S D$ in Gaussian noise added to the accumulation process; $\rho_{\lambda}^{n}$, input strength at the $\lambda$ level of difficulty (e, easy; m, medium; d, difficult) for the corresponding $n$ number of alternatives. Model variant labels abbreviate the models' structure: A, accumulator (without decay); LA, leaky accumulator (with decay); $T, t_{\text {er }}$; $C$, criterion; $e S$, equal starting point.

the proceeding run; cf. Ratcliff \& Tuerlinckx, 2002). The minimum BIC value among the values produced by these three chains is the value we report.

\section{Best-Fitting Models}

Sixteen models were fitted to the data from both Experiment 1 and Experiment 2. Table 4 shows the BIC values and their respective rankings for Experiment 1, which are data for the difficulty manipulation. Ties were awarded between BIC values that did not differ by more than 10 points; thus, one model was ranked over another only if there was very strong support $(p>.99)$ for that model, in accord with Raftery (1995, Table 6).

Inspection of Table 4 shows that models with both one nondecisional component and one decision criterion were consistently outranked by most other models, so we eliminated models of these two types from contention. Among the 12 remaining models, models with negatively correlated starting points were consistently outranked by models with identical starting points. Thus, we were left with 6 competitive models to explain the data from Experiment 1 , among which the main differences were number of parameters modeling the nondecisional component (three vs. one) and decision criterion (three vs. one).

To make sure our selection of the best-fitting model(s) would not be specific to the goodness-of-fit measure we used (viz., BIC), we refit these models to the participants' data by means of a corrected Akaike information criterion

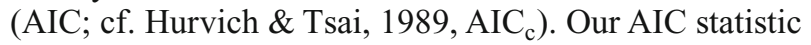
was defined by the following equation, which corresponds to Akaike's original description (e.g., Akaike, 1974) plus a bias adjustment term:

$$
\begin{aligned}
\mathrm{AIC}= & -2\left[\sum_{i} N p_{i} \ln \left(\pi_{i}\right)\right]+2 M \\
& +2 M(M+1) /(N-M-1),
\end{aligned}
$$

where $p_{i}$ and $\pi_{i}$ are the proportions of observed and predicted data in the $i$ th bin, $N$ is the number of observations in the condition, and $M$ is the number of free parameters in the model.

Parenthetically, we note that, unlike BIC estimates, AIC estimates yield no ties in ranking between two models. That is, if a model produces a lower AIC estimate than does another model, the former is deemed statistically superior to the latter regardless of the magnitude of the difference (e.g., Akaike, 1974). Table 5 shows the ranking of the six remaining models according to both BIC and AIC. Inspection of that table shows agreement between BIC and AIC for the two best-fitting models for all 4 participants and for the three best-fitting models for Participants 1 and 3 . On the basis of this agreement, we proceed to examine the fits of the three best-fitting models in Experiment 1, A( $3 T$, $1 C, e S), \mathrm{LA}(3 T, 1 C, e S)$, and $\mathrm{A}(3 T, 3 C, e S)$.

Table 6 shows mean parameter estimates for the three best-fitting models. Inspection of that table shows that, for all three of these models, (1) the change in $T_{\mathrm{er}}$ was much larger going from two to three alternatives than going from three to four alternatives, and that (2) input strength estimates decreased with increased difficulty level. The $T_{\text {er }}$ increases across number of alternatives were almost half as large for model $\mathrm{A}(3 T, 3 C, e S)$ than for the two other models (both with only one decision criterion parameter). Specifically, from two to three alternatives, there was about a 30 - versus $40-\mathrm{msec}$ increase; from three to four alternatives, there was about a 10- versus $20-\mathrm{msec}$ increase. Both magnitudes of increase are nearly linear with the logarithm of number of alternatives, and so is the increase in criterion threshold estimates for model $\mathrm{A}(3 T, 3 C, e S)$. In comparison, mean parameter estimates for competitive models with only one nondecisional parameter [e.g., $\mathrm{A}(1 T, 3 C, e S)$ in Table 7] also show that input strength estimates decreased with

Table 7

Mean Parameter Estimates for the Two Best-Fitting $1 T$ Models in Experiment 1

\begin{tabular}{lcccccccccccccccc}
\hline \multicolumn{1}{c}{ Model } & $T_{\mathrm{er}}$ & $S_{t}$ & Decay & $c^{2}$ & $c^{3}$ & $c^{4}$ & $\sigma$ & $\rho_{\mathrm{e}}^{2}$ & $\rho_{\mathrm{m}}^{2}$ & $\rho_{\mathrm{d}}^{2}$ & $\rho_{\mathrm{e}}^{3}$ & $\rho_{\mathrm{m}}^{3}$ & $\rho_{\mathrm{d}}^{3}$ & $\rho_{\mathrm{e}}^{4}$ & $\rho_{\mathrm{m}}^{4}$ & $\rho_{\mathrm{d}}^{4}$ \\
\hline $\mathrm{A}(1 T, 3 C, e S)$ & 0.356 & 0.124 & 0 & 0.577 & 0.870 & 1.004 & 0.433 & .976 & .826 & .702 & .881 & .773 & .610 & .875 & .718 & .554 \\
$\mathrm{LA}(1 T, 3 C, e S)$ & 0.353 & 0.114 & 0.175 & 0.579 & 0.841 & 0.967 & 0.460 & .974 & .859 & .724 & .920 & .795 & .615 & .905 & .763 & .602 \\
\hline
\end{tabular}

Note-Average of the parameter estimates across all 4 participants. $T_{\mathrm{er}}$, nondecision time (in seconds) for any number of alternatives; $s_{t}$, range of variability in $t_{\mathrm{er}} ; c^{n}$, criterion for the corresponding $n$ number of alternatives; $\sigma, S D$ in Gaussian noise added to the accumulation process; $\rho_{\lambda}^{n}$, input strength at the $\lambda$ level of difficulty (e, easy; m, medium; d, difficult) for the corresponding $n$ number of alternatives. Model variant labels abbreviate the models' structure: A, accumulator (without decay); LA, leaky accumulator (with decay); $T, t_{\mathrm{er}} ; C$, criterion; $e S$, equal starting point. 


\section{Experiment 1}

Model A(3T, 1C, eS)

Participant 1

Participant 2
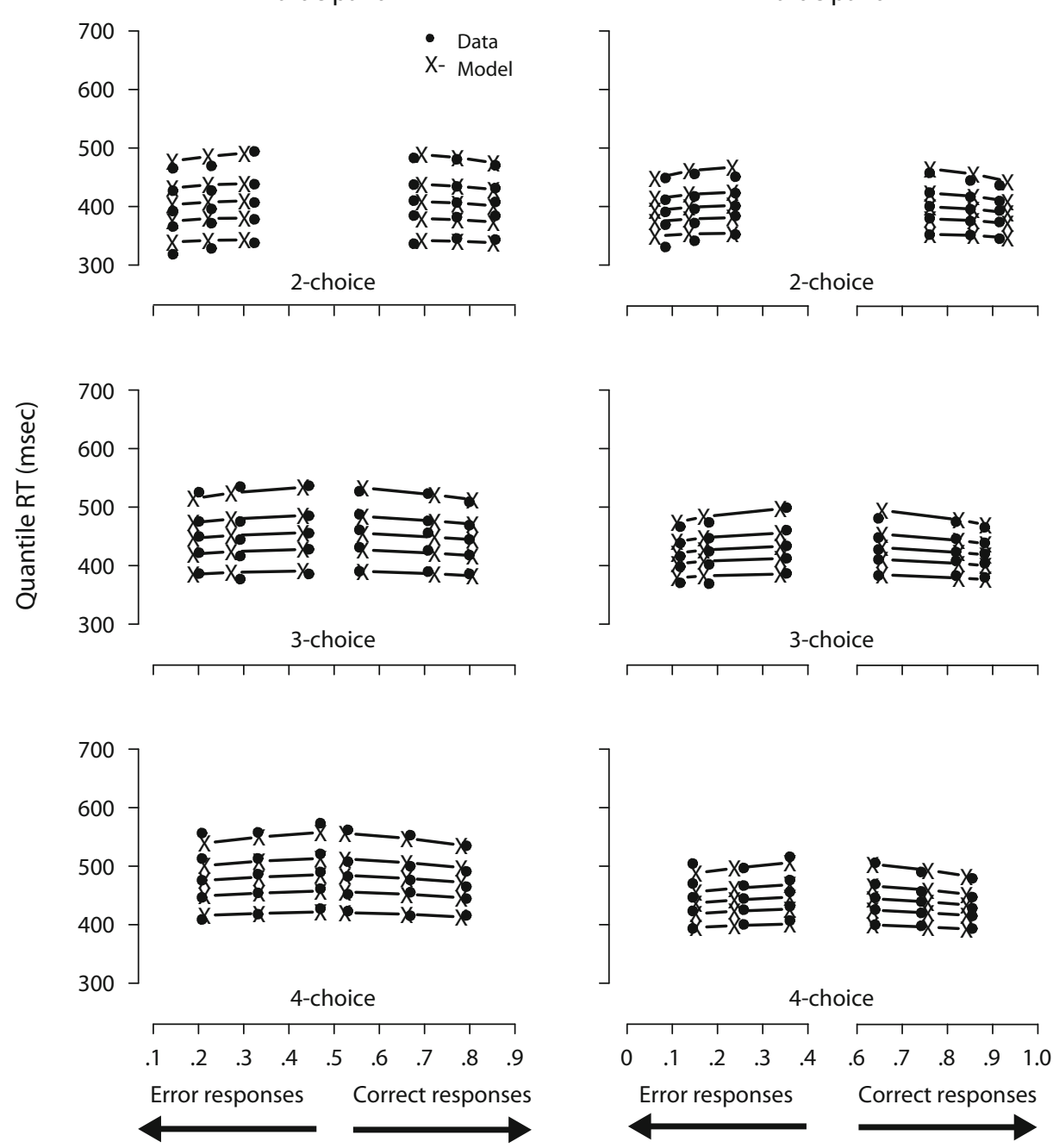

Response Probability

Figure 12. Quantile-probability plots for data from Participants 1 and 2 and predictions from the best-fitting model in Experiment 1. Reaction time (RT) points are plotted in quantile ascending order, from .1 to .9. From left to right, the three columns across error responses represent easy, medium, and difficult stimuli, followed by difficult, medium, and easy data points across correct responses. Model variant label abbreviates the model's structure: A, accumulator (without decay); $T, t_{\mathrm{er}} ; C$, criterion; $e S$, equal starting point.

increased difficulty level and that the increase in threshold criterion is nearly linear with the logarithm of number of alternatives.

With an increase in the number of alternatives, input strength parameter estimates decreased at times and increased at other times. To check how significant these changes were, we generated 25 pseudo-data-sets with 2,160 data points per condition, using average parameter estimates from model $\mathrm{A}(3 T, 1 C, e S)$, and then fit model $\mathrm{A}(3 T, 1 C, e S)$ to each of these data sets (see Table 6). The standard deviation of the mean input strength parameter estimates ranged from about 0.009 to about 0.015 . On average, two standard deviations from the mean equaled approximately 0.023 . Hence, differences between input strengths that were greater than 0.045 were deemed significant differences. Across number of alternatives, the only significant differences observed across all three models is the decrease in input strength estimates going from two to three alternatives in the difficulty condition. In addition, for model $\mathrm{A}(3 T, 3 C, e S)$, the increase in criterion estimates across number of alternatives is also nearly logarithmic.

To illustrate the fits, we plotted the data sets of all 4 participants with predictions - using BIC parameter estimates-from model $\mathrm{A}(3 T, 1 C, e S)$, the best-fitting 


\section{Experiment 1}

Model A(3T, 1C, eS)

Participant 3

Participant 4
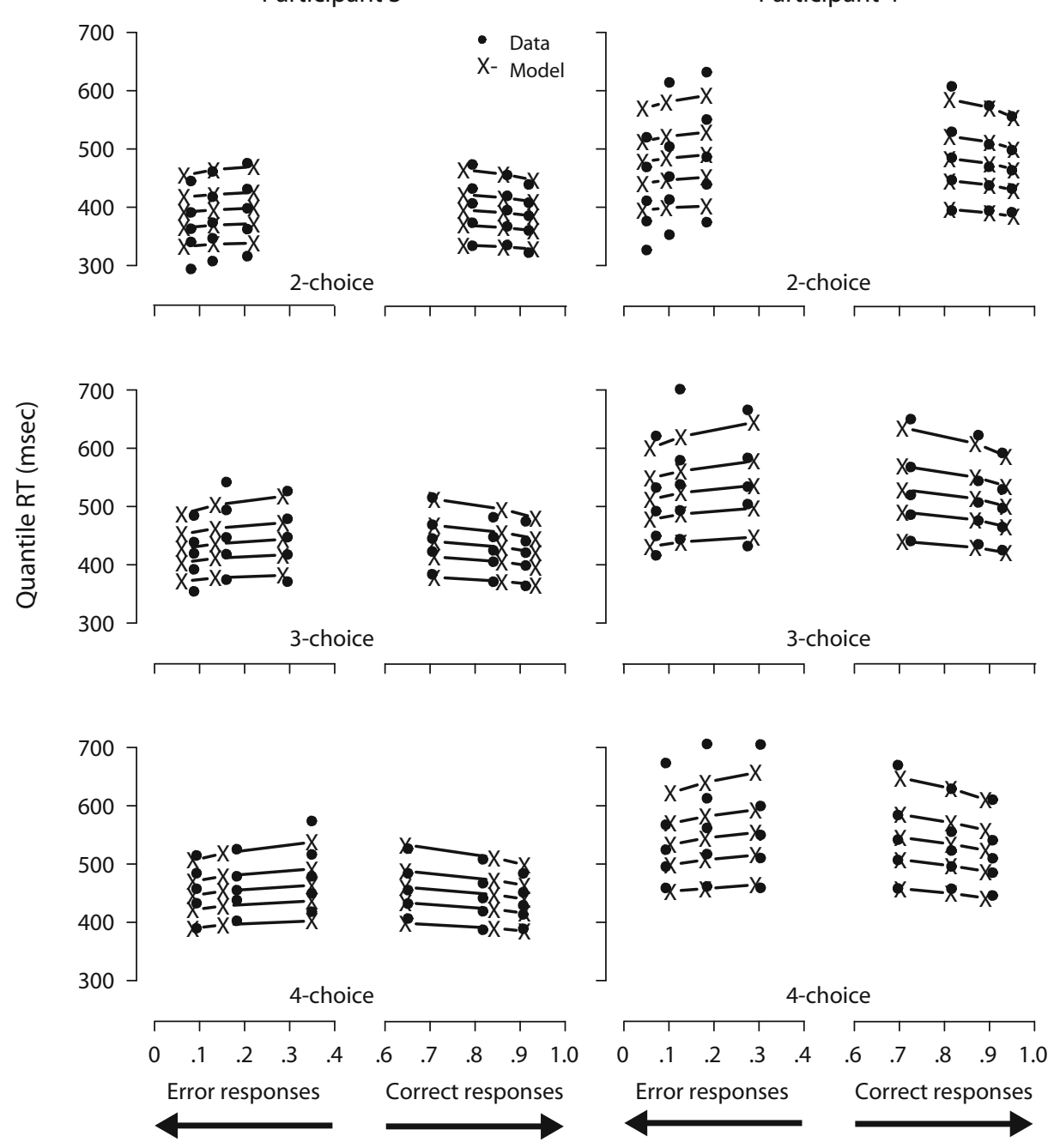

Response Probability

Figure 13. Quantile-probability plots for data from Participants 3 and 4 and predictions from the best-fitting model in Experiment 1. Reaction time (RT) points are plotted in quantile ascending order, from .1 to .9. From left to right, the three columns across error responses represent easy, medium, and difficult stimuli, followed by difficult, medium, and easy data points across correct responses. Model variant label abbreviates the model's structure: A, accumulator (without decay); $T, t_{\mathrm{er}} ; C$, criterion; $e S$, equal starting point.

model for Participants 1-3 (see Figures 12 and 13). Predictions of all three best-fitting models were very similar; hence, figures plotting their predictions were not easily distinguishable by visual inspection. Models with a decay parameter, for example, produced nearly as good fits as those without it, with decision criterion estimates $10 \%-30 \%$ lower than those for models with no decay (cf. Table 6). In addition, the two models with one nondecisional parameter (i.e., $\mathrm{A}[1 T, 3 C, e S]$ and $\mathrm{LA}[1 T, 3 C, e S]$ ) produced visually similar prediction plots as well, despite their slightly worse (quantitative) fit results. To illustrate the fits of the best-fitting models, we plotted data from Participants 1 and 2 with predictions from model
$\mathrm{LA}(3 T, 1 C, e S)$ in Figure 14 and from Participants 3 and 4 with predictions from model $\mathrm{A}(3 T, 3 C, e S)$ in Figure 15. On the basis of goodness of fit alone, we believe we cannot decide among competitive models that have the ability to mimic one another, because small differences in data may rank order them differently.

Table 8 shows the BIC values and their respective rankings for Experiment 2, which involve the proportion manipulation. Inspection of that table shows individual differences: The models that best fit Participant 5's data were different from those that best fit the data of Participants 1-3. Specifically, the competitive models for the first 3 participants were models with three nondecisional parameters and 


\section{Experiment 1}

Model LA(3T, 1C, eS)

Participant 1

Participant 2
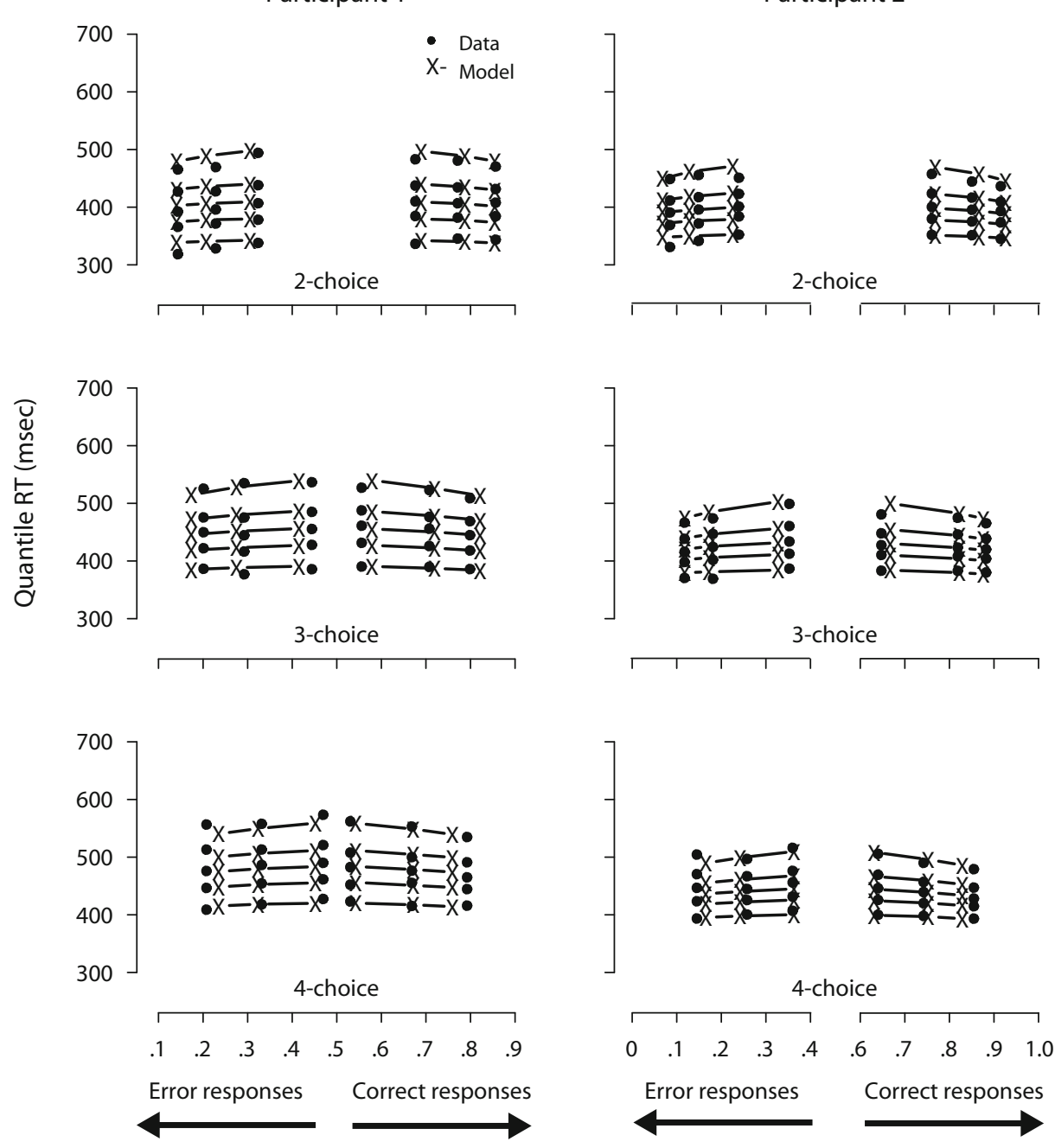

Response Probability

Figure 14. Quantile-probability plots for data from Participants 1 and 2 and predictions from the second best-fitting model in Experiment 1. Reaction time (RT) points are plotted in quantile ascending order, from .1 to .9. From left to right, the three columns across error responses represent easy, medium, and difficult stimuli, followed by difficult, medium, and easy data points across correct responses. Model variant label abbreviates the model's structure: LA, leaky accumulator (with decay); $T, t_{\mathrm{er}} ; C$, criterion; $e S$, equal starting point.

one criterion parameter, whereas Participant 5's data were better fit by models with one nondecisional parameter and one criterion parameter. For all the participants, models with equal starting points outranked models with biased starting points. Parenthetically, we did not repeat model analyses with AIC, because doing so in Experiment 1 did not alter the selection of the best-fitting models.

The best-fitting model was model $\mathrm{A}(3 T, 1 C, e S)$ for Participants $1-3$ and model $\mathrm{A}(1 T, 1 C, e S)$ for Participant 5 . Input strength estimates did not differ significantly between neutral and low-bias conditions, but they significantly increased with increases in the level of bias for the target response between low- and high-bias conditions (see
Table 9; for a comparison with low- vs. high-frequency targets, see the Appendix). Because the main manipulation in Experiment 2 involved proportion of stimuli, it may be expected that evidence parameters (i.e., input strength) do not change and that decision parameters (i.e., decision criterion) do. We could have constrained all the models that way. Rather, we decided to let the fits confirm or disconfirm this expectation, allowing both decision and input strength parameters to change across level of bias in some models (e.g., model LA[1T, 3C, eS]). If input strength parameters were estimated to be about the same values, this would allow us to constrain them to be equal. Instead, we found that decision criterion parameter estimates did not 


\section{Experiment 1}

Model A(3T, 3C, eS)

Participant 3

Participant 4
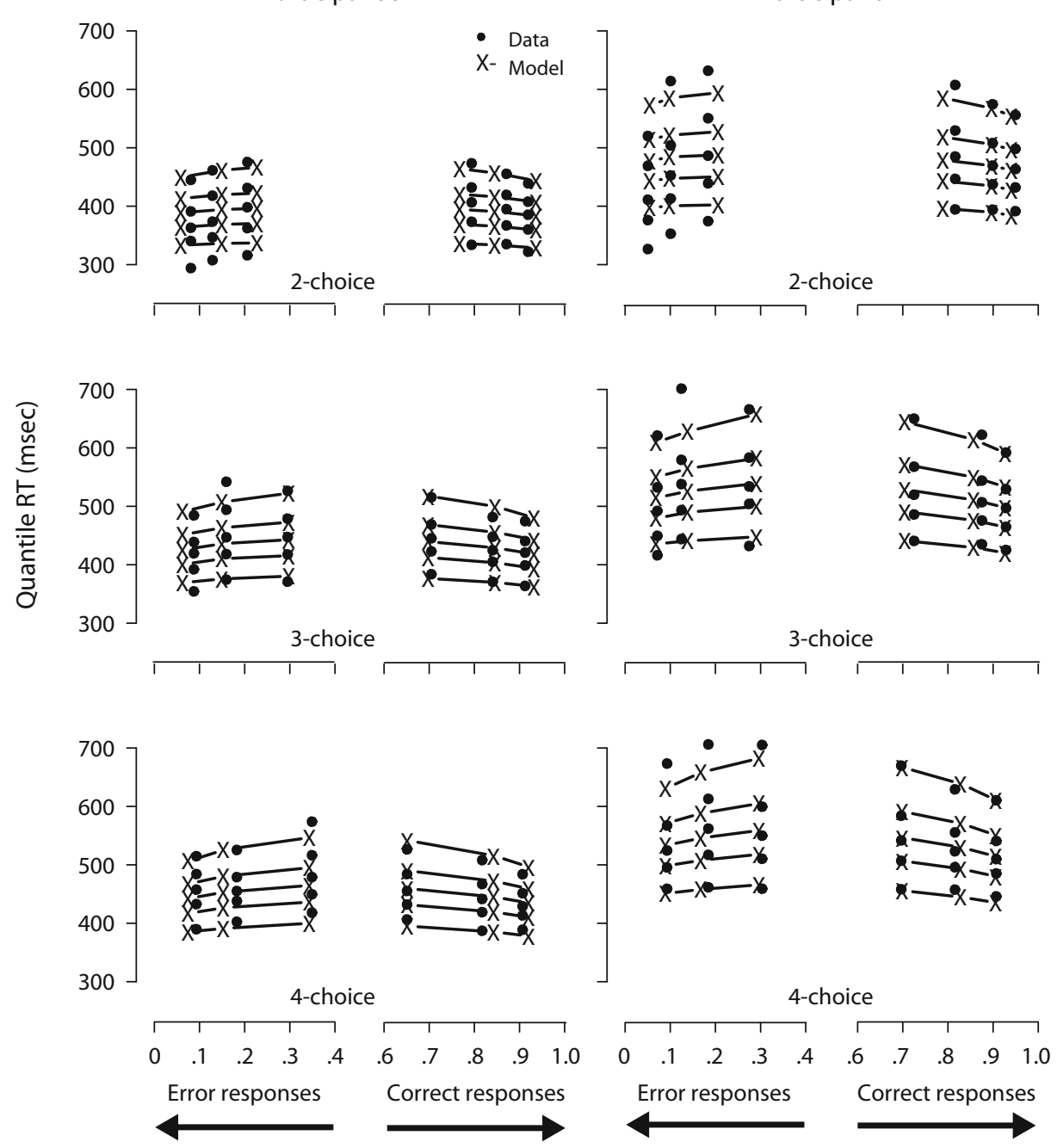

Response Probability

Figure 15. Quantile-probability plots for data from Participants 3 and 4 and predictions from the third best-fitting model in Experiment 1. Reaction time (RT) points are plotted in quantile ascending order, from .1 to .9. From left to right, the three columns across error responses represent easy, medium, and difficult stimuli, followed by difficult, medium, and easy data points across correct responses. Model variant label abbreviates the model's structure: $\mathbf{A}$, accumulator (without decay); $T, t_{\mathrm{er}} ; C$, criterion; $e S$, equal starting point.

differ much when allowed to vary across bias conditions, whereas input strength estimates differed.

This increase in target input strength estimates when target frequency is higher than distractor frequency can be interpreted as a shift in drift criterion (Gomez et al., 2007; Ratcliff, 1985; Ratcliff et al., 1999). This shift can be psychologically interpreted as a shift on the input strength dimension analogous to a change in criterion in SDT (see Ratcliff \& McKoon, 2008, Figure 3). In our case, there was a small shift in drift criterion from no bias to low bias and a larger shift from low bias to high bias. Addition of alternatives, on average, did not cause significant changes in input strength estimates for model $\mathrm{A}(3 T, 1 C, e S)$ but caused a decrease in all bias conditions for model $\mathrm{A}(1 T$, $1 C, e S)$.

Figures 16 and 17 illustrate the fits with plots of the data sets of all 4 participants with predictions from their respective best-fitting models (i.e., either $\mathrm{A}[3 T, 1 C, e S]$ or $\mathrm{A}[1 T$, $1 C, e S])$. As was the case in Experiment 1, predictions of all four best-fitting models were very similar, and, hence, their predictions were not easily distinguishable by visual inspection only. Models with decay (e.g., values around 0.5 ) produced fits similar to those for models without decay, with criterion estimates about $15 \%$ lower. 
Table 8

Bayesian Information Criterion (BIC) Rankings (Rk) for Experiment 2

\begin{tabular}{|c|c|c|c|c|}
\hline \multirow[b]{2}{*}{ Model } & \multicolumn{4}{|c|}{$\mathrm{BIC}: \mathrm{Rk}$} \\
\hline & Participant 1 & Participant 2 & Participant 3 & Participant 5 \\
\hline $\mathrm{A}(1 T, 1 C, e S)$ & $31,882: 7$ & $41,750: 15$ & $29,217: 15$ & $28,655: 1$ \\
\hline $\mathrm{A}(1 T, 1 C, b S)$ & $31,764: 4$ & $40,262: 3$ & $28,942: 10$ & $28,857: 3$ \\
\hline $\mathrm{A}(1 T, 3 C, e S)$ & $31,900: 8$ & $40,378: 6$ & $28,788: 6$ & $29,090: 11$ \\
\hline $\mathrm{A}(1 T, 3 C, b S)$ & $32,002: 11$ & $40,428: 8$ & $28,897: 8$ & $29,169: 14$ \\
\hline $\mathrm{A}(3 T, 1 C, e S)$ & $31,640: 1$ & $40,201: 1$ & $28,568: 1$ & $28,991: 7$ \\
\hline $\mathrm{A}(3 T, 1 C, b S)$ & $31,712: 2$ & $40,240: 2$ & $28,700: 3$ & $29,146: 12$ \\
\hline $\mathrm{A}(3 T, 3 C, e S)$ & $31,853: 6$ & $40,408: 7$ & $28,753: 4$ & $29,443: 16$ \\
\hline $\mathrm{A}(3 T, 3 C, b S)$ & $31,910: 8$ & $40,468: 9$ & $28,921: 9$ & $29,395: 15$ \\
\hline $\mathrm{LA}(1 T, 1 C, e S)$ & $33,048: 16$ & $41,850: 16$ & $29,389: 16$ & $28,792: 2$ \\
\hline $\mathrm{LA}(1 T, 1 C, b S)$ & $32,299: 15$ & $40,720: 14$ & $29,197: 14$ & $28,856: 3$ \\
\hline $\mathrm{LA}(1 T, 3 C, e S)$ & $32,137: 13$ & $40,583: 12$ & $28,972: 12$ & $28,853: 3$ \\
\hline $\mathrm{LA}(1 T, 3 C, b S)$ & $32,200: 14$ & $40,659: 13$ & $29,050: 13$ & $29,050: 9$ \\
\hline $\mathrm{LA}(3 T, 1 C, e S)$ & $31,736: 3$ & $40,300: 4$ & $28,647: 2$ & $28,884: 6$ \\
\hline $\mathrm{LA}(3 T, 1 C, b S)$ & $31,790: 5$ & $40,348: 5$ & $28,774: 5$ & $29,050: 9$ \\
\hline $\mathrm{LA}(3 T, 3 C, e S)$ & $31,931: 10$ & $40,510: 10$ & $28,849: 7$ & $29,001: 7$ \\
\hline $\mathrm{LA}(3 T, 3 C, b S)$ & $31,997: 11$ & $40,554: 11$ & $28,961: 11$ & $29,145: 12$ \\
\hline
\end{tabular}

Note-Ties indicate that one model did not have very strong support over the other (i.e., $p>$.99; see Raftery, 1995, Table 6). Model variant labels abbreviate the models' structure: A, accumulator (without decay); LA, leaky accumulator (with decay); $T, t_{\mathrm{er}} ; C$, criterion; $b S$, biased starting point; $e S$, equal starting point.

\section{DISCUSSION}

In this article, we explored perceptual decision making in a multiple-alternative experimental paradigm. Our aim was to examine a range of architectural features that a model could contain and attempt to determine which were needed to fit experimental data well. Several families of decision models were applied to the data from two experiments using a multiple-alternative letter discrimination task. The models assume racing diffusion processes and represent the decision process as a stochastic accumulation of evidence toward decision criteria. Statistical analysis of model fits allowed several model families to be eliminated.

The models that produce good fits for both experiments all share the following characteristics. First, the models use nine input strength parameters, one for each level of difficulty crossed with the number of alternatives. Each of the nine parameters is for a target input strength. The other (foil) input strengths are determined by the appropriate target input strength, because target and foil input strengths sum to 1 and foil input strengths are assumed to be equal. Second, accumulation of evidence is bounded to be nonnegative. Third, the models do not need lateral inhibition between accumulators. Fourth, the models do not need leakage in the accumulators.
For Experiment 1, the best-fitting models account for data with either three nondecisional parameters or three decision criteria (or both), one for each difficulty level. For Experiment 2, the best-fitting models have either one or three nondecisional parameters, but they need only one decision criterion parameter with the same value for each accumulator for two, three, and four alternatives.

In both experiments, the quality of fits for the bestfitting models cannot be discriminated by eye. These bestfitting models are all capable of fitting correct RT distributions well, but all miss the relatively short leading edges for error RT distributions obtained with two alternatives in both experiments. This mimicking among competitive models is well known (e.g., Ashby \& Townsend, 1980), and perhaps the best way to address the problem within this class of models would be to apply the models to a wider range of data and experimental paradigms (such as tasks with deadlines or response signal procedures), as suggested previously by Ratcliff (1988b).

The main architectural differences among the three best-fitting models for the perceptual difficulty manipulation involve the decay and the number of decision criterion parameters as a function of the number of alternatives. Models with a decay parameter mimic the performance of models with no decay by reducing the value of the criteria. Models with three decision criterion parameters perform

Table 9

Individual Parameter Estimates for the Best-Fitting Models in Experiment 2

\begin{tabular}{cccccccccccccccccccc}
\hline Participant & Model & $T_{\mathrm{er}}^{2}$ & $T_{\mathrm{er}}^{3}$ & $T_{\mathrm{er}}^{4}$ & $s_{t}$ & Decay & $c^{2}$ & $c^{3}$ & $c^{4}$ & $\sigma$ & $\rho_{\mathrm{n}}^{2}$ & $\rho_{\mathrm{l}}^{2}$ & $\rho_{\mathrm{h}}^{2}$ & $\rho_{\mathrm{n}}^{3}$ & $\rho_{\mathrm{l}}^{3}$ & $\rho_{\mathrm{h}}^{3}$ & $\rho_{\mathrm{n}}^{4}$ & $\rho_{\mathrm{l}}^{4}$ & $\rho_{\mathrm{h}}^{4}$ \\
\hline 1 & $\mathrm{~A}(3 T, 1 C, e S)$ & 0.269 & 0.329 & 0.352 & 0.138 & 0 & 0.723 & 0.723 & 0.723 & 0.496 & .738 & .703 & .867 & .738 & .750 & .857 & .688 & .718 & .899 \\
2 & $\mathrm{~A}(3 T, 1 C, e S)$ & 0.228 & 0.280 & 0.306 & 0.150 & 0 & 0.785 & 0.785 & 0.785 & 0.534 & .730 & .839 & .850 & .767 & .778 & .938 & .774 & .794 & .968 \\
3 & $\mathrm{~A}(3 T, 1 C, e S)$ & 0.300 & 0.335 & 0.358 & 0.106 & 0 & 0.747 & 0.747 & 0.747 & 0.352 & .745 & .802 & .839 & .758 & .793 & .808 & .785 & .832 & .892 \\
$M$ & $\mathrm{~A}(3 T, 1 C, e S)$ & 0.266 & 0.315 & 0.339 & 0.131 & 0 & 0.752 & 0.752 & 0.752 & 0.461 & .738 & .781 & .852 & .754 & .774 & .868 & .749 & .781 & .920 \\
5 & $\mathrm{~A}(1 T, 1 C, e S)$ & 0.285 & 0.285 & 0.285 & 0.178 & 0 & 1.246 & 1.246 & 1.246 & 0.235 & .672 & .658 & .726 & .513 & .525 & .576 & .418 & .433 & .524 \\
\hline
\end{tabular}

Note-Individual parameter estimates for best-fitting model, according to the Bayesian information criterion. Column labels match those in Table 6, except for $\rho_{\lambda}^{n}$, input strength at the $\lambda$ level of bias (n, neutral; 1 , low; h, high) for the corresponding $n$ number of alternatives. $M$, mean for Participants 1-3. 


\section{Experiment 2}

Model A(3T, 1C, eS)

Participant 1

Participant 2
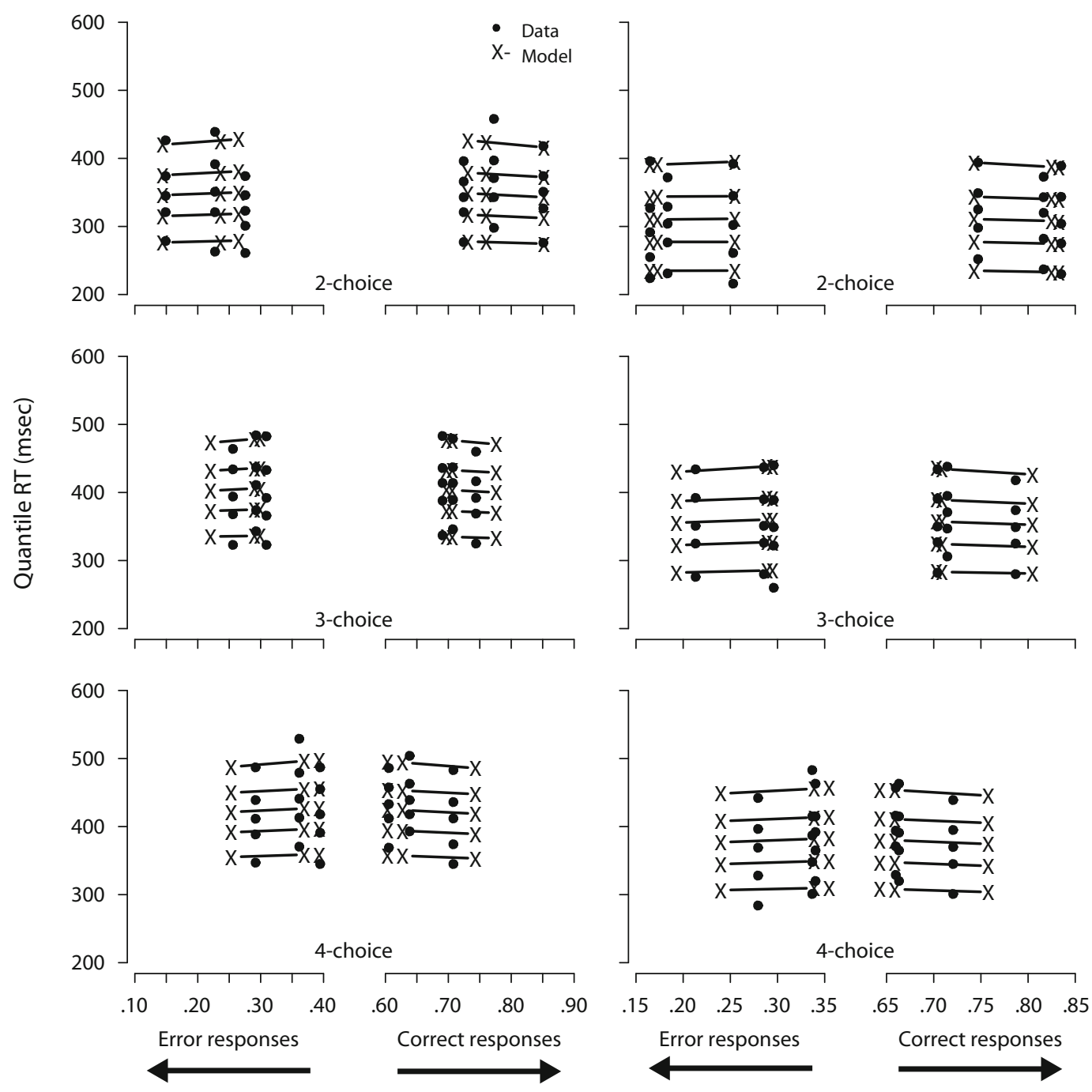

Response Probability

Figure 16. Quantile-probability plots for data from Participants 1 and 2 and predictions from the respective best-fitting model in Experiment 2. Reaction time (RT) points are plotted in quantile ascending order, from .1 to .9. From left to right, the three columns across error responses represent high-, low-, and no-bias conditions, followed by no-, low-, and high-bias data points across correct responses. Model variant label abbreviates the model's structure: $\mathrm{A}$, accumulator (without decay); $T, t_{\mathrm{er}} ; C$, criterion; $e S$, equal starting point.

like models with one criterion, with slight adjustments to the values of the criteria and also to the nondecisional component. Model analyses also show that among the three best-fitting models, irrespective of model architecture, input strength significantly decreases with the increase in number of alternatives from two to three, but only in the difficult condition. This suggests that the addition of alternatives is more disruptive when the quality of evidence is poor and that the amount of disruption that one extra alternative causes gets gradually smaller as the size of the set of alternatives gets larger.
We find that the nondecisional component of processing increases with an increase in the number of alternatives. An (ad hoc) interpretation is that addition of alternatives increases the preparation time to respond. ${ }^{5}$ The magnitude of the increase in nondecision time estimates for models with one criterion parameter, however, makes this interpretation implausible, but estimates from models with three decision criteria are consistent with it. For the latter models, parameter estimates also indicate that individuals become more cautious as the number of alternatives grows larger, and the nature of this increase in decision 


\section{Experiment 2}

Model A(3T, 1C, eS)

Participant 3
Model A(1T, 1C, eS)

Participant 5
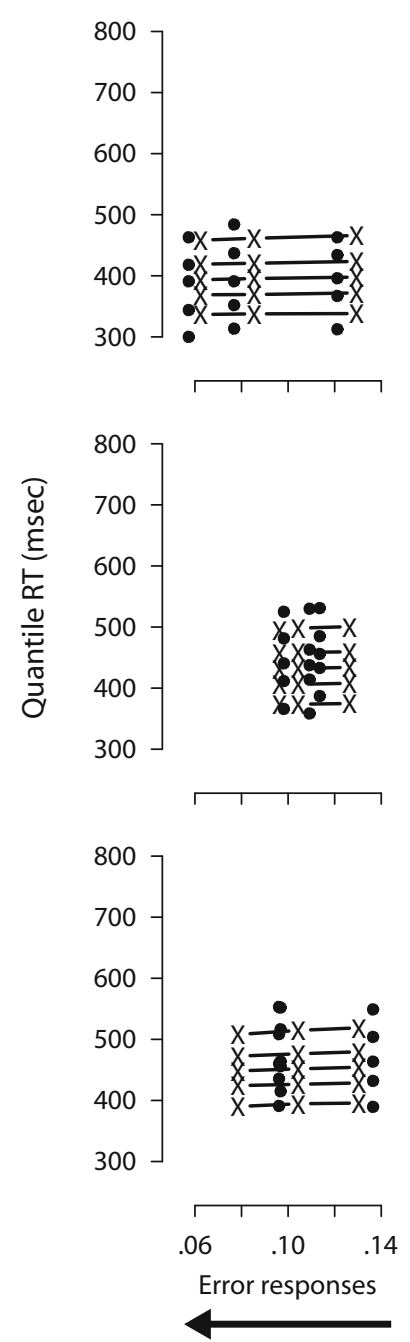
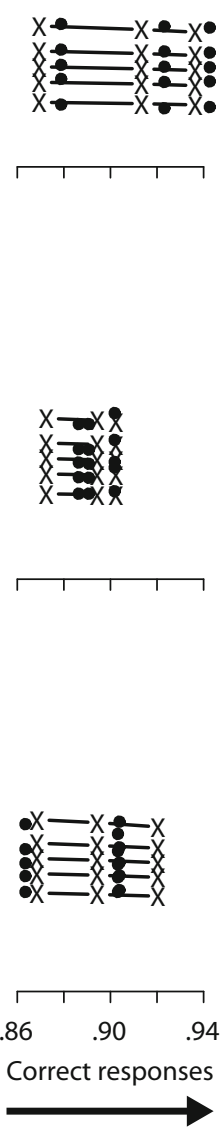
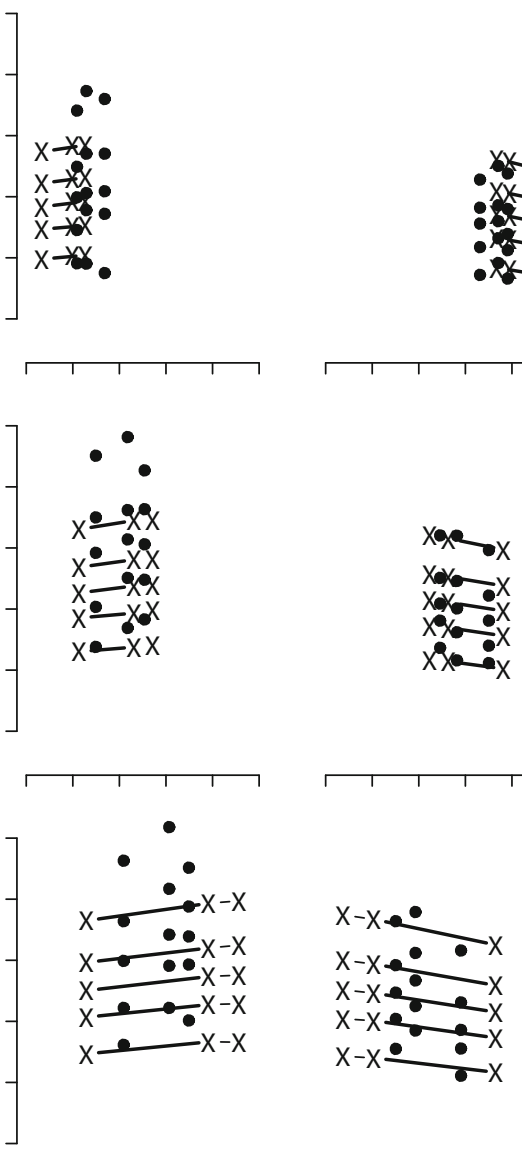

0
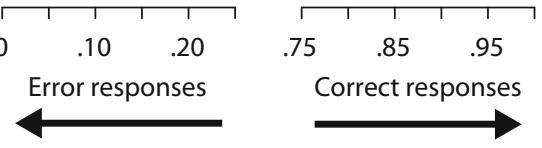

Response Probability

Figure 17. Quantile-probability plots for data from Participants 3 and 5 and predictions from the respective best-fitting models in Experiment 2. Reaction time (RT) points are plotted in quantile ascending order, from .1 to .9 . From left to right, the three columns across error responses represent high-, low-, and no-bias conditions, followed by no-, low-, and high-bias data points across correct responses. Model variant labels abbreviate the models' structures: $A$, accumulator (without decay); $T, t_{\text {er }} ; C$, criterion; $e S$, equal starting point.

criteria is nearly logarithmic. This is equivalent to Hick's law (cf. Equation 1) applied to a component of processing (as opposed to a measure of performance).

Our modeling analyses seem to lead to a conundrum. Should we select the best-fitting model strictly on numerical grounds, or may we reject the best numerical choice in favor of a model with an almost equivalent qualitative fit? For example, we prefer a model that has only one nondecisional component as a function of number of alternatives, rather than three, in order to provide an account that is more consistent with what we know about encoding and motor response subprocesses. ${ }^{6}$
Model analyses of data from Experiment 2 (which manipulated the frequency of the target stimulus) indicate that, as in Experiment 1, the nondecisional component of processing increases with an increase in the number of alternatives. Unlike in Experiment 1, the participants used the same decision criterion for two, three, and four alternatives. Both experiments give insights into how slight changes in accuracy across number of alternatives are explained: They are natural predictions of these types of models, caused by the redistribution of weight from one to two distractor accumulators and from two to three distractor accumulators. 
In summary, we describe perceptual decision making using models based on the stochastic accumulation of evidence toward a criterion. We find that several families of racing diffusion process models, differing only by a few assumptions - at times, only a single assumptioncan be discriminated when fitted to the same multiplealternative data sets. Some can be eliminated outright on the bases of statistical comparison among goodness-offit values. In the class of successful models, the models do not need lateral inhibition or decay in order to fit multiple-alternative data well. Furthermore, we find that variability in the duration of encoding and response output subprocesses and within-trial noise play important roles in the processing of evidence.

Some more general issues raised by this study are as follows. First, these are data from just one kind of experimental paradigm. It could be that the winning models are specific to this and similar paradigms, or it could be that these winning models apply over the whole range of appropriate tasks.

Second, it may seem easy to find an experimental paradigm that allows comparisons across number of alternatives. However, this is more difficult than it seems. There are problems in making sure that stimuli are equally difficult across number of alternatives, as well as making sure that reasonable ranges of accuracy and RTs are produced. A major issue for many choices of tasks is that participants might be able to adopt a strategy of, for example, first making a decision about Choices A and B, then considering C. Such strategies would result in a different class of model architectures.

Third, it may seem easy to generalize decision models from two-choice to multiple-alternative paradigms (e.g., Audley \& Pike, 1965, Figure 1; Bogacz et al., 2007; LaBerge, 1962; Laming, 1968, Figures 3.4 and 6.1; Usher \& McClelland, 2001). However, as we have noted above, there is a large number of possible assumptions that can be made about the architecture and component processes of models, and this can lead to a large family of models to evaluate.

Fourth, this strategy of competitive model testing allowed us to reduce the number of competitive modeling assumptions in the perceptual decision task we used. We suggest that this approach could be more generally useful in discriminating among families of models in other multiple-alternative paradigms (e.g., recognition memory discussed in Ratcliff \& Starns, 2009) in order to arrive at the most competitive models in those paradigms.

The research we report focused on examining theoretical descriptions of perceptual decision making that are capable of being extended from two-choice paradigms to multiple-alternative paradigms. The successful models describe the decision as a race toward a criterion driven by the accumulation of sensory evidence over time. In that dynamic accumulation of sensory evidence, we find that neither lateral inhibition between alternatives nor decay in evidence are necessary for decision making among multiple alternatives. This study also provides a case study for evaluating candidate perceptual-decision-making models.

\section{AUTHOR NOTE}

This study was supported by National Institute of Mental Health Grant R37-MH44640, awarded to R.R. Portions of this article were presented at the 48th Annual Meeting of the Psychonomic Society and at the 41st Annual Meeting of the Society for Mathematical Psychology. Correspondence concerning this article should be addressed to F. P. Leite, Department of Psychology, Ohio State University, Lima, OH 45804 (e-mail: leite.11@osu.edu).

\section{REFERENCES}

AKAIKE, H. (1974). A new look at the statistical model identification. IEEE Transactions on Automatic Control, 19, 716-723.

Albantakis, L., \& Deco, G. (2009). The encoding of alternatives in multiple-choice decision making. Proceedings of the National Academy of Sciences, 106, 10308-10313. doi:10.1073/pnas.0901621106

Ashby, F. G., \& Townsend, J. T. (1980). Decomposing the reactiontime distribution: Pure insertion and selective influence revisited. Journal of Mathematical Psychology, 21, 93-123.

Audley, R. J., \& PIKE, A. R. (1965). Some alternative stochastic models of choice. British Journal of Mathematical \& Statistical Psychology, 18, 207-225.

Bogacz, R., Usher, M., Zhang, J., \& McClelland, J. L. (2007). Extending a biologically inspired model of choice: Multi-alternatives, nonlinearity, and value-based multidimensional choice. Philosophical Transactions of the Royal Society B, 362, 1655-1670. doi:10.1098/ rstb.2007.2059

Boucher, L., Palmeri, T., Logan, G., \& Schall, J. (2007). Inhibitory control in mind and brain: An interactive race model of countermanding saccades. Psychological Review, 114, 376-397. doi:10.1037/0033 -295X.114.2.376

Brown, S., \& Heathcote, A. (2005). A ballistic model of choice response time. Psychological Review, 112, 117-128.

Brown, S., Marley, A. A. J., Donkin, C., \& Heathcote, A. (2008). An integrated model of choices and response times in absolute identification. Psychological Review, 115, 396-425. doi:10.1037/0033 $-295 X .115 .2 .396$

BusEMEYER, J. R., \& TownsEnd, J. T. (1992). Fundamental derivations from decision field theory. Mathematical Social Sciences, 23, 255282.

Busemeyer, J. R., \& Townsend, J. T. (1993). Decision field theory: A dynamic-cognitive approach to decision making in an uncertain environment. Psychological Review, 100, 432-459.

Churchland, A. K., Kiani, R., \& Shadlen, M. N. (2008). Decisionmaking with multiple alternatives. Nature Neuroscience, 11, 693-702. Available at www.nature.com/natureneuroscience.

DitTERICH, J. (2006). Stochastic models of decisions about motion direction: Behavior and physiology. Neural Networks, 19, 981-1012. doi:10.1016/j.neunet.2006.05.042

Gilmore, G. C., Hersh, H., Caramazza, A., \& Griffin, J. (1979). Multidimensional letter similarity derived from recognition errors. Perception \& Psychophysics, 25, 425-431.

Gold, J. I., \& ShadLEN, M. N. (2000). Representation of a perceptual decision in developing oculomotor commands. Nature, 404, 390-394. Available at www.nature.com.

Gomez, P., Perea, M., \& Ratcliff, R. (2007). A model of the go/no-go task. Journal of Experimental Psychology: General, 136, 389-413. doi:10.1037/0096-3445.136.3.389

Hanes, D. P., \& Schall, J. D. (1996). Neural control of voluntary movement initiation. Science, 274, 427-430.

Heekeren, H. R., Marrett, S., Ruff, D. A., Bandettini, P. A., \& UNGERLEIDER, L. G. (2006). Involvement of human left dorsolateral prefrontal cortex in perceptual decision making is independent of response modality. Proceedings of the National Academy of Sciences, 103, 10023-10028. doi:10.1073/pnas.0603949103

Hick, W. E. (1952). On the rate of gain of information. Quarterly Journal of Experimental Psychology, 4, 11-26.

Hurvich, C. M., \& TsaI, C.-L. (1989). Regression and time series model selection in small samples. Biometrika, 76, 297-307.

HyMAN, R. (1953). Stimulus information as a determinant of reaction time. Journal of Experimental Psychology, 45, 188-196. 
LaBerge, D. (1962). A recruitment theory of simple behavior. Psychometrika, 27, 375-396.

Lacouture, Y., \& Marley, A. A. J. (1995). A mapping model of bow effects in absolute identification. Journal of Mathematical Psychology, 39, 383-395.

LAMING, D. R. J. (1968). Information theory of choice-reaction times. London: Academic Press.

Link, S. (1975). The relative judgment theory of two-choice response time. Journal of Mathematical Psychology, 12, 114-135.

LUCE, R. D. (1986). Response times: Their role in inferring elementary mental organization. New York: Oxford University Press.

Mazurek, M. E., Roitman, J. D., Ditterich, J., \& Shadlen, M. N. (2003). A role for neural integrators in perceptual decision making. Cerebral Cortex, 13, 1257-1269. doi:10.1093/cercor/bhg097

McMillen, T., \& Holmes, P. (2006). The dynamics of choice among multiple alternatives. Journal of Mathematical Psychology, 50, 30-57. doi:10.1016/j.jmp.2005.10.003

Nelder, J. A., \& Mead, R. (1965). A SIMPLEX method for function minimization. Computer Journal, 7, 308-313.

Newsome, W. T., Britten, K. H., \& Movshon, J. A. (1989). Neuronal correlates of a perceptual decision. Nature, 341, 52-54

Niwa, M., \& DitTerich, J. (2008). Perceptual decisions between multiple directions of visual motion. Journal of Neuroscience, 28, 44354445. doi:10.1523/JNEUROSCI.5564-07.2008

Palmer, J., Huk, A. C., \& Shadlen, M. N. (2005). The effect of stimulus strength on the speed and accuracy of a perceptual decision. Journal of Vision, 5, 376-404.

Philiastides, M. G., Ratcliff, R., \& SAJda, P. (2006). Neural representation of task difficulty and decision-making during perceptual categorization: A timing diagram. Journal of Neuroscience, 26, 89658975. doi:10.1523/JNEUROSCI.1655-06.2006

PIKE, A. R. (1966). Stochastic models of choice behaviour: Response probabilities and latencies of finite Markov chain systems. British Journal of Mathematical \& Statistical Psychology, 19, 15-32.

RAFTERY, A. E. (1995). Bayesian model selection in social research. Sociological Methodology, 25, 111-163.

RatCliff, R. (1978). A theory of memory retrieval. Psychological Review, 85, 59-108.

RATCLIFF, R. (1981). A theory of order relation in perceptual matching. Psychological Review, 88, 552-572.

RatCLIFF, R. (1985). Theoretical interpretations of speed and accuracy of positive and negative responses. Psychological Review, 92, 215-225.

RATCLIFF, R. (1988a). Continuous versus discrete information processing: Modeling the accumulation of partial information. Psychological Review, 95, 238-255.

RatClifF, R. (1988b). A note on the mimicking of additive reaction time models. Journal of Mathematical Psychology, 32, 192-204.

RATCLIFF, R. (2002). A diffusion model account of response time and accuracy in a brightness discrimination task: Fitting real data and failing to fit fake but plausible data. Psychonomic Bulletin \& Review, 9 , 278-291.

RATCLIFF, R. (2006). Modeling response signal and response time data. Cognitive Psychology, 53, 195-237. doi:10.1016/j.cogpsych .2005.10.002

Ratcliff, R., Cherian, A., \& Segraves, M. (2003). A comparison of macaque behavior and superior colliculus neuronal activity to predictions from models of two-choice decisions. Journal of Neurophysiology, 90, 1392-1407.

Ratcliff, R., Gomez, P., \& McKoon, G. (2004). Diffusion model account of lexical decision. Psychological Review, 111, 159-182.

Ratcliff, R., Hasegawa, Y. T., Hasegawa, Y. P., Smith, P. L., \& SeGRAVES, M. A. (2007). Dual diffusion model for single-cell recording data from the superior colliculus in a brightness-discrimination task. Journal of Neurophysiology, 97, 1756-1774. doi:10.1152/ jn.00393.2006

Ratcliff, R., \& McKoon, G. (2008). The diffusion decision model: Theory and data for two-choice decision tasks. Neural Computation, 20, 873-922.

Ratcliff, R., Philiastides, M. G., \& Sajda, P. (2009). Quality of evidence for perceptual decision making is indexed by trial-to-trial variability of the EEG. Proceedings of the National Academy of Sciences, 106, 6539-6544. doi:10.1073/pnas.0812589106
RATCLIFF, R., \& Rouder, J. F. (1998). Modeling response times for twochoice decisions. Psychological Science, 9, 347-356.

Ratcliff, R., \& Rouder, J. F. (2000). A diffusion model account of masking in two-choice letter identification. Journal of Experimental Psychology: Human Perception \& Performance, 26, 127-140.

Ratcliff, R., \& Smith, P. L. (2004). A comparison of sequential sampling models for two-choice reaction time. Psychological Review, 111, 333-367.

Ratcliff, R., \& Starns, J. J. (2009). Modeling confidence and response time in recognition memory. Psychological Review, 116, 5983. doi: $10.1037 / \mathrm{a} 0014086$

Ratcliff, R., Thapar, A., \& McKoon, G. (2006). Aging, practice, and perceptual tasks: A diffusion model analysis. Psychology \& Aging, 21, 353-371

Ratcliff, R., Thapar, A., Smith, P. L., \& McKoon, G. (2005). Aging and response times: A comparison of sequential sampling models. In J. Duncan, P. McLeod, \& L. Phillips (Eds.), Measuring the mind: Speed, control, and age (pp. 3-32). Oxford: Oxford University Press.

RATCLIFF, R., \& TUERLINCKX, F. (2002). Estimating parameters of the diffusion model: Approaches to dealing with contaminant reaction times and parameter variability. Psychonomic Bulletin \& Review, 9, 438-481.

Ratcliff, R., \& Van Dongen, H. P. A. (2009). Sleep deprivation affects multiple distinct cognitive processes. Psychonomic Bulletin \& Review, 16, 742-751.

Ratcliff, R., Van Zandt, T., \& McKoon, G. (1999). Connectionist and diffusion models of reaction time. Psychological Review, 106, 261-300.

Roe, R. M., Busemeyer, J. R., \& Townsend, J. T. (2001). Multialternative decision field theory: A dynamic connectionist model of decision making. Psychological Review, 108, 370-392.

Roitman, J. D., \& Shadlen, M. N. (2002). Response of neurons in the lateral intraparietal area during a combined visual discrimination reaction time task. Journal of Neuroscience, 22, 9475-9489.

Salzman, C. D., \& Newsome, W. T. (1994). Neural mechanisms for forming a perceptual decision. Science, 264, 231-237. Available at www.jstor.org/stable/2883370.

Schwarz, G. (1978). Estimating the dimension of a model. Annals of Statistics, 6, 461-464.

Shadlen, M. N., \& Newsome, W. T. (2001). Neural basis of a perceptual decision in the parietal cortex (area lip) of the rhesus monkey. Journal of Neurophysiology, 86, 1916-1936. Available at http://jn.physiology.org.

Smith, G. A., \& CAREW, M. (1987). Decision time unmasked: Individuals adopt different strategies. Australian Journal of Psychology, 39, 339-351

SMITH, P. L. (1995). Psychophysically principled models of simple visual reaction time. Psychological Review, 102, 567-593.

SMITH, P. L. (2000). Stochastic dynamic models of response time and accuracy: A foundational primer. Journal of Mathematical Psychology, 44, 408-463.

Smith, P. L., \& RatclifF, R. (2009). An integrated theory of attention and decision making in visual signal detection. Psychological Review, 116, 283-317. doi:10.1037/a0015156

Smith, P. L., Ratcliff, R., \& Wolfgang, B. J. (2004). Attention orienting and the time course of perceptual decisions: Response time distributions with masked and unmasked displays. Vision Research, 44, 1297-1320.

Smith, P. L., \& Van Zandt, T. (2000). Time-dependent Poisson counter models of response latency in simple judgment. British Journal of Mathematical \& Statistical Psychology, 53, 293-315.

Smith, P. L., \& VicKers, D. (1988). The accumulator model of twochoice discrimination. Journal of Mathematical Psychology, 32, 135-168.

Supèr, H., Spekreisse, H., \& Lamme, V. A. F. (2001). Two distinct modes of sensory processing observed in monkey primary visual cortex (v1). Nature Neuroscience, 4, 304-310. Available at http:// neurosci.nature.com.

SwENSSON, R. G. (1972). The elusive trade-off: Speed versus accuracy in visual discrimination tasks. Perception \& Psychophysics, 12, 16-32.

Swets, J. A., TANner, W. P., JR., \& Birdsall, T. G. (1961). Decision processes in perception. Psychological Review, 68, 301-340.

Thapar, A., Ratcliff, R., \& McKoon, G. (2003). A diffusion model 
analysis of the effects of aging on letter discrimination. Psychology \& Aging, 18, 415-429.

Thomas, R. D. (2006). Processing time predictions of current models of perception in the classic additive factors paradigm. Journal of Mathematical Psychology, 50, 441-455. doi:10.1016/j.jmp.2006.05.006

Townsend, J. T., \& AshBY, F. G. (1983). The stochastic modeling of elementary psychological processes. Cambridge: Cambridge University Press.

Usher, M., \& MCClelland, J. L. (2001). On the time course of perceptual choice: The leaky competing accumulator model. Psychological Review, 108, 550-592.

Usher, M., \& McClelland, J. L. (2004). Loss aversion and inhibition in dynamical models of multialternative choice. Psychological Review, 111, 759-769.

Usher, M., Olami, Z., \& McClelland, J. L. (2002). Hick's law in a stochastic race model with speed-accuracy tradeoff. Journal of Mathematical Psychology, 46, 704-715.

VICKERS, D. (1970). Evidence for an accumulator of psychophysical discrimination. Ergonomics, 13, 37-58.

Vickers, D., Caudrey, D., \& Willson, R. (1971). Discriminating between frequency of occurrence of two alternative events. Acta Psychologica, 35, 151-172.

WASSERMAN, L. (2000). Bayesian model selection and model averaging. Journal of Mathematical Psychology, 44, 92-107.

WelFord, A. T. (ED.) (1980). Reaction times. London: Academic Press.

\section{NOTES}

1. For exceptions that were published, online or in print, after the original submission date of a previous version of this article, see Albantakis and Deco (2009) and Churchland, Kiani, and Shadlen (2008). Churchland et al. used a motion discrimination task to study two- and four-choice situations and found that there might exist a neural deadline for the decision and that four alternatives require more evidence accumulation than do two alternatives. Albantakis and Deco used a similar paradigm and found evidence for a physiological advantage of a pooled, multineuronal representation of choice alternatives.
2. The data from Experiment 2 contained conditions (viz., low and high bias) in which stimuli were not equally probable. For these conditions (center and right panels under Experiment 2 in Figure 5), Hick's law should be expected to predict MRT only if $\log (n+1)$ in Equation 1 were replaced by $\sum_{i=0}^{n} p_{i} \log \left(1 / p_{i}\right)$, where $p_{i}$ is the probability of each response choice (cf. Usher et al., 2002). For our data, Equation 1 was a good enough approximation, so we used it for simplicity of exposition.

3. Individual cutoffs (lower, upper) are as follows (in milliseconds). For two, three, and four alternatives, respectively, in Experiment 1: Participant $1,(240,650),(300,850)$, and $(320,950)$; Participant 2, (280, $600),(300,650),(300,650)$; Participant $3,(250,1,000),(300,1,000)$, (330, 1,000); Participant 4, (240, 900), (240, 1,100), (240, 1,200).

4. Individual cutoffs (lower, upper) are as follows (in milliseconds). For two, three, or four alternatives, respectively, in Experiment 2: Participant 1, (200, 700), (240, 700), (290, 700); Participant 2, $(200,700)$, $(250,700),(280,750)$; Participant $3,(230,700),(300,800),(310,820)$; Participant 5, (220, 650), (270,650), (270,650).

5 . We find evidence to support an adjustment in the nondecisional component due to an increase in the number of alternatives. Data from the other 4 participants in an experiment in which the only manipulated variable was number of alternatives (with 7,616 observations) show that the leading edge (i.e., .1 quantile) of the correct RT distribution increases, on average, by approximately $24 \mathrm{msec}$ from two to three alternatives and by approximately $12 \mathrm{msec}$ from three to four alternatives. Even after familiarizing themselves with the stimulus-response mapping, it is plausible that participants require extra time getting set to make their decision because of their knowledge of being in a condition with more possible responses. We termed the time needed for this psychological adjustment preparation time.

6 . Such a model is feasible with only one free parameter accounting for nondecision time for two alternatives and with a fixed increase from two to three and from three to four alternatives. In fitting such a model (using $T_{\mathrm{er}}+15[\mathrm{msec}]$ and $T_{\mathrm{er}}+22[\mathrm{msec}]$ for three and four alternatives, respectively) to data from Experiment 1, we find that an alternate $\mathrm{A}(1 T *, 3 C, e S)$ model challenges the best-fitting model for Participants $1-3$ and becomes the best-fitting model for Participant 4 . 


\section{APPENDIX \\ Low- Versus High-Frequency Targets}

As an anonymous reviewer pointed out, a more curious reader might wonder whether a model analysis that pitched low-frequency versus high-frequency targets could provide extra information. We do not present such an analysis in the main text, because the conditions with low-frequency targets (in low- or high-bias conditions) have considerably fewer data points than do the conditions with high-frequency targets. Thus, modeling results for low-frequency targets alone might not be as meaningful as we would like them to be.

In the main text, we report results from an aggregate analysis, considering both low- and high-frequency target responses, which, because of the discrepancy in the number of data points we highlight above, are dominated by the high-frequency responses. We foresaw that the magnitude of the effects might be amplified should we report only on the basis of high-frequency targets, for example, but we avoided that option so as not to bias our analysis. Nevertheless, Tables A1 and A2 are included here to serve as an illustration of how input strength and criterion parameters might change across the frequency of the target and how much the parameter estimates might change from the aggregate to the specific. (To produce Tables A1 and A2, we used the best-fitting models shown in Table 9 so that a comparison would be straightforward.)

Taking the means row of Table A1 and comparing it with the means row of Table 9, we note that the decision criterion is about $96 \%$ of the overall estimate among high-frequency targets and that the increase in input strength estimates is more prominent across all bias levels. Both observations are consistent with the participants' expectations of the high-frequency targets: Psychologically, expected targets might need to accumulate less evidence before a decision in their favor is made (although this difference is not quantitatively significant in our case), and the more a target is expected, the larger its strength to elicit an identification response.

Inspecting Table A2 in the same way and comparing its means row with the means row of Table 9, we note that the decision criterion is about 3\% higher than the overall estimate and that input strength estimates show a decreasing trend with increase in bias toward other targets (essentially biasing participants against these low-frequency targets). These observations are again consistent with participants' expectations of the high-frequency targets: More evidence might be required of targets that appear infrequently than of targets that appear frequently (although again, it is the case that this difference is not quantitatively significant), and the less a target is expected, the smaller its strength to elicit an identification response. Together, these two tables confirm the top-down influence on the perceptual task caused by the expectation of the stimulus, which is opposite in nature to the bottom-up influence caused by the perceptual difficulty manipulation in Experiment 1 .

Table A1

Individual Parameter Estimates in Experiment 2: High-Frequency

\begin{tabular}{|c|c|c|c|c|c|c|c|c|c|c|c|c|c|c|c|c|c|c|c|}
\hline Participant & Model & $T_{\mathrm{er}}^{2}$ & $T_{\mathrm{er}}^{3}$ & $T_{\mathrm{er}}^{4}$ & $s_{t}$ & Decay & $c^{2}$ & $c^{3}$ & $c^{4}$ & $\sigma$ & $\rho_{\mathrm{n}}^{2}$ & $\rho_{1}^{2}$ & $\rho_{\mathrm{h}}^{2}$ & $\rho_{\mathrm{n}}^{3}$ & $\rho_{1}^{3}$ & $\rho_{\mathrm{h}}^{3}$ & $\rho_{\mathrm{n}}^{4}$ & $\rho_{1}^{4}$ & $\rho_{\mathrm{h}}^{4}$ \\
\hline 1 & $\mathrm{~A}(3 T, 1 C, e S)$ & 0.265 & 0.323 & 0.351 & 0.133 & 0 & 0.726 & 0.726 & 0.726 & 0.456 & .701 & .761 & .904 & .671 & .772 & .894 & .643 & .756 & .960 \\
\hline 2 & & 235 & 0.283 & 0.311 & 0. & 0 & 0.687 & 0.687 & 0.687 & 0.427 & .675 & .856 & .869 & .680 & .803 & .924 & .667 & .713 & .955 \\
\hline 3 & $T, 1 C, e S)$ & 0.298 & 0.334 & 0.352 & 0.106 & 0 & 0.744 & 0.744 & 0.744 & 0.328 & .723 & .779 & .817 & .743 & .782 & .808 & .746 & .853 & 852 \\
\hline$M$ & & 266 & & & & & & & & & .700 & .799 & .863 & .698 & .786 & .875 & .685 & .774 & .922 \\
\hline 5 & $\mathrm{~A}(1 T, 1 C, e S)$ & 0.329 & 0.329 & 0.329 & 0.272 & 0 & 1.048 & 1.048 & 1.048 & 0.162 & .747 & .773 & .876 & .571 & .643 & .771 & .501 & .576 & .736 \\
\hline
\end{tabular}

Note-Individual parameter estimates for high-frequency targets. Column labels match those of Table 6 , except for $\rho_{\lambda}^{\mathrm{n}}$, input strength at the $\lambda$ level of bias (n, neutral; 1 , low; h, high) for the corresponding $n$ number of alternatives. $M$, mean for Participants $1-3$.

Table A2

Individual Parameter Estimates in Experiment 2: Low-Frequency

\begin{tabular}{cccccccccccccccccccc}
\hline Participant & Model & $T_{\mathrm{er}}^{2}$ & $T_{\mathrm{er}}^{3}$ & $T_{\mathrm{er}}^{4}$ & $s_{t}$ & Decay & $c^{2}$ & $c^{3}$ & $c^{4}$ & $\sigma$ & $\rho_{\mathrm{n}}^{2}$ & $\rho_{1}^{2}$ & $\rho_{\mathrm{h}}^{2}$ & $\rho_{\mathrm{n}}^{3}$ & $\rho_{1}^{3}$ & $\rho_{\mathrm{h}}^{3}$ & $\rho_{\mathrm{n}}^{4}$ & $\rho_{\mathrm{l}}^{4}$ & $\rho_{\mathrm{h}}^{4}$ \\
\hline 1 & $\mathrm{~A}(3 T, 1 C, e S)$ & 0.265 & 0.336 & 0.368 & 0.139 & 0 & 0.811 & 0.811 & 0.811 & 0.639 & .818 & .523 & .626 & .857 & .793 & .793 & .860 & .810 & .807 \\
2 & $\mathrm{~A}(3 T, 1 C, e S)$ & 0.240 & 0.294 & 0.329 & 0.148 & 0 & 0.743 & 0.743 & 0.743 & 0.588 & .771 & .748 & .605 & .866 & .774 & .755 & .886 & .901 & .778 \\
3 & $\mathrm{~A}(3 T, 1 C, e S)$ & 0.303 & 0.342 & 0.361 & 0.112 & 0 & 0.758 & 0.758 & 0.758 & 0.371 & .772 & .863 & .859 & .819 & .863 & .767 & .827 & .781 & .839 \\
$M$ & $\mathrm{~A}(3 T, 1 C, e S)$ & 0.269 & 0.324 & 0.353 & 0.133 & 0 & 0.771 & 0.771 & 0.771 & 0.533 & .787 & .711 & .697 & .847 & .810 & .772 & .858 & .831 & .808 \\
5 & $\mathrm{~A}(1 T, 1 C, e S)$ & 0.353 & 0.353 & 0.353 & 0.254 & 0 & 0.974 & 0.974 & 0.974 & 0.153 & .788 & .718 & .659 & .588 & .581 & .545 & .511 & .489 & .518 \\
\hline
\end{tabular}

Note-Individual parameter estimates for low-frequency targets. Column labels match those of Table 6 , except for $\rho_{\lambda}^{\mathrm{n}}$, input strength at the $\lambda$ level of bias (n, neutral; 1, low; h, high) for the corresponding $n$ number of alternatives. $M$, mean for Participants $1-3$. 